TOKYO J. MATH.

VoL. 9, No. 1, 1986

\title{
On the Mixed Problem for Wave Equation in a Domain with a Corner
}

\section{Masaru TANIGUCHI}

(Communicated by K. Kojima)

\section{Introduction}

The purpose of this paper is to generalize the results in [5] and to obtain the complete results.

We consider mixed problems

( I )

and

$$
\begin{aligned}
& \left\{\begin{array}{l}
L_{1}[u]=\frac{\partial^{2} u}{\partial t^{2}}-\frac{\partial^{2} u}{\partial x^{2}}-\frac{\partial^{2} u}{\partial y^{2}}+d u=f(t, x, y) \\
u(0, x, y)=u_{0}(x, y), \quad u_{t}(0, x, y)=u_{1}(x, y) \\
\left.B_{1}[u]\right|_{x=0}=\left.\left(\frac{\partial u}{\partial x}+b \frac{\partial u}{\partial y}-c \frac{\partial u}{\partial t}+\alpha u\right)\right|_{x=0}=g_{1}(t, y) \\
\left.B_{2}[u]\right|_{y=0}=\left.\left(\frac{\partial u}{\partial y}+\frac{1}{b} \frac{\partial u}{\partial x}-\frac{c}{b} \frac{\partial u}{\partial t}+\frac{\alpha}{b} u\right)\right|_{y=0}=g_{2}(t, x) \\
(t, x, y) \in\left(\boldsymbol{R}_{+}^{1}\right)^{3}
\end{array}\right. \\
& \left\{\begin{array}{l}
L_{1}[u]=f(t, x, y) \\
u(0, x, y)=u_{0}(x, y), \quad u_{t}(0, x, y)=u_{1}(x, y) \\
\left.B_{3}[u]\right|_{x=0}=\left.\left(\frac{\partial u}{\partial x}+\alpha u\right)\right|_{x=0}=g_{1}(t, y) \\
\left.B_{4}[u]\right|_{y=0}=\left.\left(\frac{\partial u}{\partial y}+\beta u\right)\right|_{y=0}=g_{2}(t, x) \\
(t, x, y) \in\left(\boldsymbol{R}_{+}^{1}\right)^{3}
\end{array}\right.
\end{aligned}
$$

$\quad\left\{\begin{array}{l}L_{2}[u]=\frac{\partial^{2} u}{\partial t^{2}}-\frac{\partial^{2} u}{\partial x^{2}}-\frac{\partial^{2} u}{\partial y^{2}}-\frac{\partial^{2} u}{\partial z^{2}}+d u=f(t, x, y, z) \\ u(0, x, y, z)=u_{0}(x, y, z), \quad u_{t}(0, x, y, z)=u_{1}(x, y, z) \\ \left.B_{5}[u]\right|_{x=0}=\left.\left(\frac{\partial u}{\partial x}+\alpha u\right)\right|_{x=0}=g_{1}(t, y, z)\end{array}\right.$

Received September 7, 1984 


$$
\left\{\begin{array}{l}
\left.B_{6}[u]\right|_{y=0}=\left.\left(\frac{\partial u}{\partial y}+\beta u\right)\right|_{y=0}=g_{2}(t, x, z) \\
\left.B_{7}[u]\right|_{z=0}=\left.\left(\frac{\partial u}{\partial z}+\gamma u\right)\right|_{z=0}=g_{3}(t, x, y) \\
(t, x, y, z) \in\left(R_{+}^{1}\right)^{4}
\end{array}\right.
$$

where $b, c, d, \alpha, \beta$ and $\gamma$ are complex constants.

In [5], for the problem (I), we obtained the result that the problem (I) is $L^{2}$-well-posed if mixed problems

$$
\left\{\begin{array}{l}
L_{1}[u]=f(t, x, y) \\
u(0, x, y)=u_{0}(x, y), \quad u_{t}(0, x, y)=u_{1}(x, y) \\
\left.B_{1}[u]\right|_{x=0}=g_{1}(t, y) \\
(t, x, y) \in\left(R_{+}^{1}\right)^{2} \times R^{1}
\end{array}\right.
$$

and

$$
\left\{\begin{array}{l}
L_{1}[u]=f(t, x, y) \\
u(0, x, y)=u_{0}(x, y), \quad u_{t}(0, x, y)=u_{1}(x, y) \\
\left.B_{2}[u]\right|_{y=0}=g_{2}(t, x) \\
(t, x, y) \in R_{+}^{1} \times R^{1} \times R_{+}^{1}
\end{array}\right.
$$

are $L^{2}$-well-posed and $b \neq \pm i$. In this paper, we shall show the result that the problem (I) is $L^{2}$-well-posed if the problems (1) and (2) are $L^{2}$ well-posed and $b= \pm i$. Therefore, we get the complete result that the problem (I) is $L^{2}$-well-posed if and only if the problems (1) and (2) are $L^{2}$-well-posed. Also, in [5], for the problems (II) and (III), we were concerned with the mixed problems with homogenenous boundary condition and could not obtain the boundary estimate for the solution. In this paper, we treat the mixed problems with non-homogeneous boundary condition and get the similar energy inequality as to the one for the mixed problem with the Neumann boundary condition in a domain with smooth boundary (see [3]).

To obtain the energy inequality, we reduce the mixed problem for wave equation to the one for symmetric hyperbolic system of first order with non-negative boundary condition. This method was used in [1], [4], [5], [6] and [7].

An outline of this paper is as follows. In $\S 1$, we explain the notation. In $\S 2$, we state the results. In $\S 3$, we are concerned with roots of the quadratic equation $(c+1) z^{2}+2 b z+(c-1)=0 \quad(b= \pm i)$. In $\S 4$, we treat the mixed problem for symmetric hyperbolic system of first order 
in a domain with a corner and give the simple proof comparing with the one in [5]. In $\S 5$, we obtain the energy inequality. In $\S 6$ and $\S 7$, we prove the existence of the classical solution.

\section{§ 1. Notation.}

$\boldsymbol{R}^{n}\left(\boldsymbol{C}^{n}\right): n$-dimensional real (complex) Euclidean space.

$\boldsymbol{R}_{+}^{n} \quad$ : the set $\left\{(x, y) \mid x>0, y \in \boldsymbol{R}^{n-1}\right\}$.

$$
\begin{aligned}
& \|u\|_{m, \mu, T}^{2}=\sum_{\alpha+\beta+\gamma+\delta=m} \int_{0}^{T} d t \int_{0}^{\infty} d x \int_{0}^{\infty} d y\left|e^{-\mu t} \mu^{\alpha}\left(\frac{\partial}{\partial t}\right)^{\beta}\left(\frac{\partial}{\partial x}\right)^{r}\left(\frac{\partial}{\partial y}\right)^{\delta} u\right|^{2} \text { or } \\
& \sum_{\alpha+\beta+\gamma+\delta+\theta=m} \int_{0}^{T} d t \int_{0}^{\infty} d x \int_{0}^{\infty} d y \int_{0}^{\infty} d z\left|e^{-\mu t} \mu^{\alpha}\left(\frac{\partial}{\partial t}\right)^{\beta}\left(\frac{\partial}{\partial x}\right)^{\gamma}\left(\frac{\partial}{\partial y}\right)^{\delta}\left(\frac{\partial}{\partial z}\right)^{\theta} u\right|^{2} . \\
& \langle u\rangle_{m, \mu, T}^{2}=\sum_{\alpha+\beta+\gamma=m} \int_{0}^{T} d t \int_{0}^{\infty} d y\left|e^{-\mu t} \mu^{\alpha}\left(\frac{\partial}{\partial t}\right)^{\beta}\left(\frac{\partial}{\partial y}\right)^{\gamma} u\right|^{2} \quad \text { or } \\
& \sum_{\alpha+\beta+\gamma+\delta=m} \int_{0}^{T} d t \int_{0}^{\infty} d y \int_{0}^{\infty} d z\left|e^{-\mu t} \mu^{\alpha}\left(\frac{\partial}{\partial t}\right)^{\beta}\left(\frac{\partial}{\partial y}\right)^{\gamma}\left(\frac{\partial}{\partial z}\right)^{\delta} u\right|^{2} . \\
& \langle u\rangle_{m, \mu, T}^{2}=\sum_{\alpha+\beta+\gamma=m} \int_{0}^{T} d t \int_{0}^{\infty} d x\left|e^{-\mu t} \mu^{\alpha}\left(\frac{\partial}{\partial t}\right)^{\beta}\left(\frac{\partial}{\partial x}\right)^{r} u\right|^{2} \quad \text { or } \\
& \sum_{\alpha+\beta+\gamma+\delta=m} \int_{0}^{T} d t \int_{0}^{\infty} d x \int_{0}^{\infty} d z\left|e^{-\mu t} \mu^{\alpha}\left(\frac{\partial}{\partial t}\right)^{\beta}\left(\frac{\partial}{\partial x}\right)^{r}\left(\frac{\partial}{\partial z}\right)^{\delta} u\right|^{2} . \\
& \langle\langle u\rangle\rangle_{m, \mu, T}^{2}=\sum_{\alpha+\beta+\gamma+\delta=m} \int_{0}^{T} d t \int_{0}^{\infty} d x \int_{0}^{\infty} d y\left|e^{-\mu t} \mu^{\alpha}\left(\frac{\partial}{\partial t}\right)^{\beta}\left(\frac{\partial}{d x}\right)^{T}\left(\frac{\partial}{\partial y}\right)^{\delta} u\right|^{2} \text {. } \\
& \|\| u(t) \|_{m, \mu}^{2}=\sum_{\alpha+\beta+\gamma+\delta=m} \int_{0}^{\infty} d x \int_{0}^{\infty} d y\left|e^{-\mu t} \mu^{\alpha}\left(\frac{\partial}{\partial t}\right)^{\beta}\left(\frac{\partial}{\partial x}\right)^{\gamma}\left(\frac{\partial}{\partial y}\right)^{\delta} u\right|^{2} \quad \text { or } \\
& \sum_{\alpha+\beta+\gamma+\delta+\theta=m} \int_{0}^{\infty} d x \int_{0}^{\infty} d y \int_{0}^{\infty} d z\left|e^{-\mu t} \mu^{\alpha}\left(\frac{\partial}{\partial t}\right)^{\beta}\left(\frac{\partial}{\partial x}\right)^{\gamma}\left(\frac{\partial}{\partial y}\right)^{\delta}\left(\frac{\partial}{\partial z}\right)^{\theta} u\right|^{2} .
\end{aligned}
$$

(, ) : the inner product in $L^{2}\left[\left(\boldsymbol{R}_{+}^{1}\right)^{2}\right]$ or $L^{2}\left[\left(\boldsymbol{R}_{+}^{1}\right)^{8}\right]$.

$(()$,$) : the inner product in C^{j}$.

$\langle u, v\rangle=\int_{0}^{\infty} \int_{0}^{\infty} u \bar{v} d y d z$ or $\int_{0}^{\infty} u \bar{v} d y$.

$\langle u, v\rangle=\int_{0}^{\infty} \int_{0}^{\infty} u \bar{v} d x d z$ or $\int_{0}^{\infty} u \bar{v} d x$.

$\langle\langle u, v\rangle\rangle=\int_{0}^{\infty} \int_{0}^{\infty} u \bar{v} d x d y$.

$[u, v]=\int_{-\infty}^{\infty} u \bar{v} d \eta$.

$H_{m}(\Omega):$ the Sobolev space.

$\mathscr{\mathcal { C }}_{m, \mu}\left[\left(\boldsymbol{R}_{+}^{1}\right)^{n}\right]:$ the space of functions which are obtained by the com- 
pletion of $C_{0}^{\infty}\left[\left(\overline{\boldsymbol{R}}_{+}^{1}\right)^{n}\right]$ with the norm $\|u\|_{m, \mu, \infty}$.

$\Lambda_{x, \mu}^{-\theta}=\bar{\mho}_{x}\left(\xi^{2}+\mu^{2}\right)^{-\theta / 2} \mho_{x}$, etc. .

$T_{\eta, \mu}^{-\theta}(\cdot)=\left(\eta^{2}+\mu^{2}\right)^{-\theta / 2} \times(\cdot)$, etc. .

$D_{x}=\frac{\partial}{\partial x}$, etc. .

§2. Statement of the result.

We consider the mixed problems (I), (II) and (III).

We assume following conditions for the problem (I):

$$
b=i \text { or } b=-i
$$

and

(C.2) The quadratic equation

$$
(c+1) z^{2}+2 b z+(c-1)=0
$$

has roots in the domain $\bar{D}=\{z \in C|| z \mid \leqq 1, \operatorname{Re} z \leqq 0\}$ if they are different and in $D=\{z \in C|| z \mid<1, \operatorname{Re} z<0\}$ if they are equal.

Definition 1. (i) We say that $\left\{f, g_{1}, u_{0}, u_{1}\right\}$ satisfies the compatibility condition of order $k$ in the region $\Omega_{1}\left(\Omega_{2}\right)$ if the following condition $\left(\mathrm{C}_{1 k}\right)$ holds:

$$
\begin{aligned}
\widetilde{B}^{(m)}\left(f, u_{0}, u_{1}\right) & \left.\equiv \sum_{j=0}^{m}\left\{\widetilde{B}_{1 j}^{(m)} u_{j}\right\}\right|_{x=0} \\
& =\left.\left(D_{t}^{m-1} g_{1}\right)\right|_{t=0} \quad(m=1,2, \cdots, k)
\end{aligned}
$$

where

$$
\left\{\begin{array}{l}
\sum_{j=0}^{m} \widetilde{B}_{1 j}^{(m)} D_{t}^{j} u \equiv D_{t}^{m-1}\{\widetilde{B} u\} \\
u_{2+i} \equiv\left\{\left.\left(D_{t}^{i} f\right)\right|_{t=0}-\left(D_{t}^{i} \widetilde{L}-D_{t}^{2+i}\right) u\right\} \quad(i=0,1,2, \cdots) \\
\widetilde{L}=L_{1} \text { or } L_{2}, \quad \widetilde{B}=B_{1} \text { or } B_{8} \text { or } B_{8}
\end{array}\right.
$$

and

$$
\left\{\begin{array}{l}
\Omega_{1}=\{y \mid y \geqq 0\} \text { or }\{(y, z) \mid y \geqq 0, z \geqq 0\} \\
\Omega_{2}=\left\{y \mid y \in R^{1}\right\} .
\end{array}\right.
$$

(ii) We say that $\left\{f, g_{2}, u_{0}, u_{1}\right\}$ satisfies the compatibility condition of order $k$ in the region $\Omega_{3}\left(\Omega_{4}\right)$ if the following condition $\left(\mathrm{C}_{2 \mathrm{k}}\right)$ holds: 
$\left(\mathrm{C}_{2 k}\right)$

$$
\begin{aligned}
\widetilde{B}^{(m)}\left(f, u_{0}, u_{1}\right) & \left.\equiv \sum_{j=0}^{m}\left\{\widetilde{B}_{2 j}^{(m)} u_{j}\right\}\right|_{y=0} \\
& =\left.\left(D_{t}^{m-1} g_{2}\right)\right|_{t=0} \quad(m=1,2, \cdots k)
\end{aligned}
$$

where

$$
\left\{\begin{array}{l}
\sum_{j=0}^{m} \widetilde{B}_{2 j}^{(m)} D_{t}^{j} u=D_{t}^{m-1}(\widetilde{B} u) \\
u_{2+i} \equiv\left\{\left.\left(D_{t}^{i} f\right)\right|_{t=0}-\left(D_{t}^{i} \widetilde{L}-D_{t}^{2+\imath}\right) u\right\} \quad(i=0,1,2, \cdots) \\
\widetilde{L}=L_{1} \text { or } L_{2}, \quad \widetilde{B}=B_{2} \text { or } B_{4} \text { or } B_{6}
\end{array}\right.
$$

and

$$
\left\{\begin{array}{l}
\Omega_{3}=\{x \mid x \geqq 0\} \text { or }\{(x, z) \mid x \geqq 0, z \geqq 0\} \\
\Omega_{4}=\left\{x \mid x \in R^{1}\right\} .
\end{array}\right.
$$

(iii) We say that $\left\{f, g_{3}, u_{0}, u_{1}\right\}$ satisfies the compatibility condition of order $k$ in the region $\Omega_{5}$ if the following condition $\left(\mathrm{C}_{s k}\right)$ holds:

$\left(\mathrm{C}_{s k}\right)$

$$
\begin{aligned}
\widetilde{B}^{(m)}\left(f, u_{0}, u_{1}\right) & \left.\equiv \sum_{j=0}^{m}\left\{\widetilde{B}_{3 j}^{(m)} u_{j}\right\}\right|_{z=0} \\
& =\left.\left(D_{t}^{m-1} g_{s}\right)\right|_{t=0} \quad(m=1,2, \cdots, k)
\end{aligned}
$$

where

$$
\left\{\begin{array}{l}
\sum_{j=0}^{m} \widetilde{B}_{3 j}^{(m)} D_{t}^{j} u \equiv D_{t}^{m-1}(\widetilde{B} u) \\
u_{2+i} \equiv\left\{\left.\left(D_{t}^{i} f\right)\right|_{t=0}-\left(D_{t}^{i} \tilde{L}-D_{t}^{2+i}\right) u\right\} \quad(i=0,1,2, \cdots) \\
\widetilde{L}=L_{2}, \quad \widetilde{B}=B_{7}
\end{array}\right.
$$

and

$$
\Omega_{\mathrm{b}}=\{(x, y) \mid x \geqq 0, y \geqq 0\} .
$$

Definition 2. (i) We say that $\left\{g_{1}, g_{2}\right\}$ satisfies the compatibility condition $\left(\mathrm{D}_{k}\right)(k=1,3,5)$ if the following condition holds:

$$
\begin{aligned}
\left(\mathrm{D}_{1}\right) & g_{1}(t, 0) & =b \cdot g_{2}(t, 0) \\
\left(\mathrm{D}_{8}\right) & b g_{2 x x}(t, 0) & =\left[\left(\frac{\partial^{2}}{\partial t^{2}}-\frac{\partial^{2}}{\partial y^{2}}+d\right) g_{1}\right](t, 0)-\left(B_{1} f\right)(t, 0,0) \\
\left(\mathrm{D}_{\mathrm{s}}\right) & b g_{2 x x x x}(t, 0) & =\left[\left(\frac{\partial^{2}}{\partial t^{2}}-\frac{\partial^{2}}{\partial y^{2}}+d\right)^{2} g_{1}\right](t, 0)-B_{1}\left(f_{t t}-f_{y y}+f_{x x}+d f\right)(t, 0,0) .
\end{aligned}
$$

(ii) We say that $\left\{g_{1}, g_{2}\right\}$ satisfies the compatibility condition $\left(\mathrm{I}_{k}\right)$ $(k=1,3,5,7,9)$ if the following condition holds:

$\left(\mathrm{I}_{1}\right)$

$$
\left.\left(\frac{\partial}{\partial y}+\beta\right) g_{1}\right|_{y=0}=\left.\left(\frac{\partial}{\partial x}+\alpha\right) g_{2}\right|_{x=0}
$$


(I $\left.\mathrm{I}_{3}\right)\left.\quad\left(\frac{\partial}{\partial y}+\beta\right)\left(\frac{\partial}{\partial y}\right)^{2} g_{1}\right|_{y=0}=\left.\left(\frac{\partial}{\partial x}+\alpha\right)(M+d) g_{2}\right|_{x=0}-\left.\left(\frac{\partial}{\partial x}+\alpha\right)\left(\frac{\partial}{\partial y}+\beta\right) f\right|_{x=y=0}$

(I $\left.\mathrm{I}_{\mathrm{b}}\right)\left.\quad\left(\frac{\partial}{\partial y}+\beta\right)\left(\frac{\partial}{\partial y}\right)^{4} g_{1}\right|_{y=0}=\left.\left(\frac{\partial}{\partial x}+\alpha\right)(M+d)^{2} g_{2}\right|_{z=0}$

$$
-\left.\left(\frac{\partial}{\partial x}+\alpha\right)\left(\frac{\partial}{\partial y}+\beta\right)\left(M+\frac{\partial^{2}}{\partial y^{2}}+d\right) f\right|_{x=y=0}
$$

(I $\left.\mathrm{I}_{7}\right)\left.\quad\left(\frac{\partial}{\partial y}+\beta\right)\left(\frac{\partial}{\partial y}\right)^{6} g_{1}\right|_{y=0}=\left.\left(\frac{\partial}{\partial x}+\alpha\right)(M+d)^{8} g_{2}\right|_{z=0}-\left(\frac{\partial}{\partial x}+\alpha\right)\left(\frac{\partial}{\partial y}+\beta\right)$

$$
\times\left.\left[(M+d)^{2}+\frac{\partial^{2}}{\partial y^{2}}\left\{M+\frac{\partial^{2}}{\partial y^{2}}+d\right\}\right] f\right|_{x=y=0}
$$

$\left(\mathrm{I}_{8}\right)$

$$
\begin{aligned}
\left.\left(\frac{\partial}{\partial y}+\beta\right)\left(\frac{\partial}{\partial y}\right)^{8} g_{1}\right|_{y=0}= & \left.\left(\frac{\partial}{\partial x}+\alpha\right)(M+d)^{4} g_{2}\right|_{x=0} \\
& -\left(\frac{\partial}{\partial x}+\alpha\right)\left(\frac{\partial}{\partial y}+\beta\right)\left[(M+d)^{3}+\frac{\partial^{2}}{\partial y^{2}}(M+d)^{2}\right. \\
& \left.+\frac{\partial^{4}}{\partial y^{4}}\left\{M+\frac{\partial^{2}}{\partial y^{2}}+d\right\}\right]\left.f\right|_{x=y=0}
\end{aligned}
$$

where

$$
M=\frac{\partial^{2}}{\partial t^{2}}-\frac{\partial^{2}}{\partial x^{2}} \quad \text { or } \quad M=\frac{\partial^{2}}{\partial t^{2}}-\frac{\partial^{2}}{\partial x^{2}}-\frac{\partial^{2}}{\partial y^{2}}
$$

(iii) We say that $\left\{g_{2}, g_{8}\right\}$ satisfies the compatibility condition $\left(\mathrm{II}_{k}\right)$ $(k=1,3,5)$ if the following condition holds:

$$
\begin{aligned}
\left.\left(\frac{\partial}{\partial z}+\gamma\right) g_{2}\right|_{z=0}= & \left.\left(\frac{\partial}{\partial y}+\beta\right) g_{3}\right|_{y=0} \\
\left.\left(\frac{\partial}{\partial z}+\gamma\right) g_{2 s s}\right|_{z=0}= & \left.\left(\frac{\partial}{\partial y}+\beta\right)\left(\frac{\partial^{2}}{\partial t^{2}}-\frac{\partial^{2}}{\partial x^{2}}-\frac{\partial^{2}}{\partial y^{2}}+d\right) g_{3}\right|_{y=0} \\
& -\left.\left(\frac{\partial}{\partial y}+\beta\right)\left(\frac{\partial}{\partial z}+\gamma\right) f\right|_{y=s=0} \\
\left.\left(\frac{\partial}{\partial z}+\gamma\right) g_{2 s z z z}\right|_{z=0}= & \left.\left(\frac{\partial}{\partial y}+\beta\right)\left(\frac{\partial^{2}}{\partial t^{2}}-\frac{\partial^{2}}{\partial x^{2}}-\frac{\partial^{2}}{\partial y^{2}}+d\right)^{2} g_{3}\right|_{y=0} \\
& -\left.\left(\frac{\partial}{\partial y}+\beta\right)\left(\frac{\partial}{\partial z}+\gamma\right)\left(\frac{\partial^{2}}{\partial t^{2}}-\frac{\partial^{2}}{\partial x^{2}}-\frac{\partial^{2}}{\partial y^{2}}+\frac{\partial^{2}}{\partial z^{2}}+d\right) f\right|_{y=z=0^{0}}
\end{aligned}
$$

(iv) We say that $\left\{g_{1}, g_{3}\right\}$ satisfies the compatibility condition $\left(\operatorname{III}_{k}\right)$ $(k=1,3,5)$ if the following condition holds:

$\left(\mathrm{III}_{1}\right)$

$$
\left.\left(\frac{\partial}{\partial x}+\alpha\right) g_{8}\right|_{x=0}=\left.\left(\frac{\partial}{\partial z}+\gamma\right) g_{1}\right|_{s=0}
$$


$\left.\left(\mathrm{III}_{3}\right) \quad\left(\frac{\partial}{\partial x}+\alpha\right) g_{3 x x}\right|_{x=0}=\left.\left(\frac{\partial}{\partial z}+\gamma\right)\left(\frac{\partial^{2}}{\partial t^{2}}-\frac{\partial^{2}}{\partial y^{2}}-\frac{\partial^{2}}{\partial z^{2}}+d\right) g_{1}\right|_{z=0}$

$$
-\left.\left(\frac{\partial}{\partial x}+\alpha\right)\left(\frac{\partial}{\partial z}+\gamma\right) f\right|_{x=z=0}
$$

$\left.\left(\mathrm{III}_{8}\right) \quad\left(\frac{\partial}{\partial x}+\alpha\right) g_{3 x x x x}\right|_{x=0}=\left.\left(\frac{\partial}{\partial z}+\gamma\right)\left(\frac{\partial^{2}}{\partial t^{2}}-\frac{\partial^{2}}{\partial y^{2}}-\frac{\partial^{2}}{\partial z^{2}}+d\right)^{2} g_{1}\right|_{z=0}$

$$
-\left.\left(\frac{\partial}{\partial x}+\alpha\right)\left(\frac{\partial}{\partial z}+\gamma\right)\left(\frac{\partial^{2}}{\partial t^{2}}-\frac{\partial^{2}}{\partial y^{2}}-\frac{\partial^{2}}{\partial z^{2}}+\frac{\partial^{2}}{\partial x^{2}}+d\right) f\right|_{\varepsilon=s=0} .
$$

Definition 3. (i) We say that $\left\{f, g_{1}, u_{0}, u_{1}\right\}$ has the property $\left(\mathrm{E}_{k}\right)$ :

$\left(\mathrm{E}_{k}\right) \quad\left\{f, g_{1}, u_{0}, u_{1}\right\}$ satisfies the $\left(\mathrm{C}_{1 k}\right)$ in $\Omega_{1}=\{y \mid y \geqq 0\}$ and has an extension $\left\{\widetilde{f}, \widetilde{g}_{1}, \widetilde{u}_{0}, \widetilde{u}_{1}\right\}$ which satisfies the $\left(\mathrm{C}_{1 k}\right)$ in $\Omega_{2}$ and has the same regularity as $\left\{f, g_{1}, u_{0}, u_{1}\right\}$.

(ii) We say that $\left\{f, g_{2}, u_{0}, u_{1}\right\}$ has the property $\left(\mathrm{E}_{k}^{\prime}\right)$ :

( $\left.\mathrm{E}_{k}^{\prime}\right) \quad\left\{f, g_{2}, u_{0}, u_{1}\right\}$ satisfies the $\left(\mathrm{C}_{2 k}\right)$ in $\Omega_{3}=\{x \mid x \geqq 0\}$ and has an extension $\left\{\widetilde{f}, \widetilde{g}_{2}, \widetilde{u}_{0}, \tilde{u}_{1}\right\}$ which satisfies the $\left(\mathrm{C}_{2 k}\right)$ in $\Omega_{4}$ and has the same regularity as $\left\{f, g_{2}, u_{0}, u_{1}\right\}$.

(iii) We say that $\left\{\left\{f, g_{1}, u_{0}, u_{1}\right\},\left\{f, g_{2}, u_{0}, u_{1}\right\}\right\}$ has the property ( $\left.\mathrm{E}_{k}^{\prime \prime}\right)$ :

$\left(\mathrm{E}_{k}^{\prime \prime}\right)$ (1) $\left\{f, g_{1}, u_{0}, u_{1}\right\}$ satisfies the $\left(\mathrm{C}_{1 k}\right)$ in $\{(y, z) \mid y \geqq 0, z \geqq 0\}$ and has an extension $\left\{\widetilde{f}, \widetilde{g}_{1}, \widetilde{u}_{0}, \widetilde{u}_{1}\right\}$ which satisfies the $\left(\mathrm{C}_{1 k}\right)$ in $\{(y, z) \mid y \geqq 0, z \in$ $\left.\boldsymbol{R}^{1}\right\}$ and has the same regularity as $\left\{f, g_{1}, u_{0}, u_{1}\right\}$.

(2) $\left\{f, g_{2}, u_{0}, u_{1}\right\}$ satisfies the $\left(\mathrm{C}_{2 k}\right)$ in $\{(x, z) \mid x \geqq 0, z \geqq 0\}$ and has an extension $\left\{\widetilde{f}, \widetilde{g}_{2}, \widetilde{u}_{0}, \widetilde{u}_{1}\right\}$ which satisfies the $\left(C_{2 k}\right)$ in $\left\{(x, z) \mid(x, z) \in \boldsymbol{R}^{2}\right\}$ and has the same regularity as $\left\{f, g_{2}, u_{0}, u_{1}\right\}$.

(3) $\left\{\widetilde{g}_{1}, \widetilde{g}_{2}\right\}$ satisfies the compatibility conditions $\left(\mathrm{I}_{2 j-1}\right)(j=1,2, \cdots$, $\left.\left[\frac{k-2}{2}\right]\right)$.

We now state our results,

Theorem 1. Assume the conditions (C.1) and (C.2). Let $u$ be the solusion of the problem (I) which belongs to $\mathscr{H}_{2, \mu}\left[\left(\boldsymbol{R}_{+}^{1}\right)^{8}\right]$. Then, there exist positive constants $C$ and $\mu_{0}$ such that the following inequality holds for any $t \in \boldsymbol{R}_{+}^{1}$ and any $\mu \geqq \mu_{0}$

$$
\begin{aligned}
& \|\| u(t)\left\|_{1, \mu}^{2}+\mu\right\| u \|_{1, \mu, t}^{2} \\
& \quad+\mu \sum_{k=0}^{1}\left\{\left\langle\Lambda_{\nu, \mu}^{-1 / 2}\left(\frac{\partial}{\partial x}\right)^{k} u\right\rangle_{1-k, \mu, t}^{2}+\left\langle\left\langle\Lambda_{x, \mu}^{-1 / 2}\left(\frac{\partial}{\partial y}\right)^{k} u\right\rangle\right\rangle_{1-k, \mu, t}^{2}\right\}
\end{aligned}
$$




$$
\leqq C\left\{\|u(0)\|_{1, \mu}^{2}+\frac{1}{\mu}\|f\|_{0, \mu, t}^{2}+\frac{1}{\mu}\left\langle\Lambda_{\nu, \mu}^{1 / 2} g_{1}\right\rangle_{0, \mu, t}^{2}+\frac{1}{\mu}\left\langle\left\langle\Lambda_{x, \mu}^{1 / 2} g_{2}\right\rangle_{0, \mu, t}^{2}\right\}\right. \text {. }
$$

THEOREM 2. Assume the conditions (C.1) and (C.2). Let $\left(f, g_{1}, g_{2}, u_{0}, u_{1}\right)$ belongs to $C_{0}^{\infty}\left[\left(\overline{\boldsymbol{R}}_{+}^{1}\right)^{8}\right] \times\left[C_{0}^{\infty}\left[\left(\overline{\boldsymbol{R}}_{+}^{1}\right)^{2}\right]\right]^{4}$ and suppose that the conditions $\left(\mathrm{E}_{\odot}^{\prime}\right),\left(\mathrm{C}_{10}\right)$ in $\{y \mid y \geqq 0\},\left(D_{1}\right)$, $\left(\mathrm{D}_{\mathrm{s}}\right)$ and $\left(\mathrm{D}_{\mathrm{s}}\right)$ hold.

Then, there exists a unique classical solution $u \in \mathscr{H}_{b, \mu}\left[\left(\boldsymbol{R}_{+}^{1}\right)^{8}\right]$ of the problem (I) which satisfies (2.2).

REMARK 1. We have Theorem 2 by the assumption that the conditions $\left(\mathrm{E}_{8}\right),\left(\mathrm{C}_{28}\right)$ in $\{x \mid x \geqq 0\},\left(\mathrm{D}_{1}\right),\left(\mathrm{D}_{8}\right)$ and $\left(\mathrm{D}_{8}\right)$ hold.

THEOREM 3. Let $u$ be the solution of the problem (II) which belongs to $\mathscr{\mathscr { C }}_{2, \mu}\left[\left(\boldsymbol{R}_{+}^{1}\right)^{2}\right]$.

Then, there exist positive constants $C$ and $\mu_{0}$ such that the following inequality holds for any $t \in R_{+}^{1}$ and any $\mu \geqq \mu_{0}$

$$
\begin{aligned}
& \|u(t)\|_{1, \mu}^{2}+\mu\|u\|_{1, \mu, t}^{2} \\
& +\mu \sum_{k=0}^{1}\left\{\left\langle\Lambda_{, \mu}^{-1 / 2}\left(\frac{\partial}{\partial x}\right)^{k} u\right\rangle_{1-k, \mu, t}^{2}+\left\langle\left\langle\Lambda_{x, \mu}^{-1 / 2}\left(\frac{\partial}{\partial y}\right)^{k} u\right\rangle_{1-k, \mu, t}^{2}\right\}\right. \\
& \quad \leqq C\left\{\|u(0)\|_{1, \mu}^{2}+\frac{1}{\mu}\|f\|_{0, \mu, t}^{2}+\frac{1}{\mu}\left\langle\Lambda_{y, \mu}^{1 / 2} g_{1}\right\rangle_{0, \mu, t}^{2}+\frac{1}{\mu}\left\langle\left\langle\Lambda_{x, \mu}^{1 / 2} g_{2}\right\rangle_{0, \mu, t}^{2}\right\}\right.
\end{aligned}
$$

THEOREM 4. Let $\left(f, g_{1}, g_{2}, u_{0}, u_{1}\right)$ belongs $C_{0}^{\infty}\left[\left(\overline{\boldsymbol{R}}_{+}^{1}\right)^{8}\right] \times\left[C_{0}^{\infty}\left[\left(\overline{\boldsymbol{R}}_{+}^{1}\right)^{2}\right]\right]^{4}$ and suppose that the conditions $\left(\mathrm{E}_{8}^{\prime}\right),\left(\mathrm{C}_{18}\right)$ in $\{y \mid y \geqq 0\}\left(\mathrm{I}_{1}\right),\left(\mathrm{I}_{8}\right)$ and $\left(\mathrm{I}_{8}\right)$ hold.

Then, there exists a unique classical solution $u \in \mathscr{H}_{s, \mu}\left[\left(\boldsymbol{R}_{+}^{1}\right)^{8}\right]$ of the problem (II) which satisfies (2.3).

REMARK 2. We have Theorem 4 by the assumption that the conditions $\left(\mathrm{E}_{8}\right),\left(\mathrm{C}_{28}\right)$ in $\{x \mid x \geqq 0\},\left(\mathrm{I}_{1}\right),\left(\mathrm{I}_{8}\right)$ and $\left(\mathrm{I}_{8}\right)$ hold.

THEOREM 5. Let $u$ be the solution of the problem (III) which belongs to $\mathscr{H}_{2, \mu}\left[\left(\boldsymbol{R}_{+}^{1}\right)^{4}\right]$.

Then, there exist positive constants $C$ and $\mu_{0}$ such that the following inequality holds for any $t \in R_{+}^{1}$ and any $\mu \geqq \mu_{0}$

$$
\begin{aligned}
& \|\| u(t)\left\|_{1, \mu}^{2}+\mu\right\| u \|_{1, \mu, t}^{2}+\mu \sum_{k=0}^{1}\left\{\left\langle\Lambda_{y, \varepsilon, \mu}^{-1 / 2}\left(\frac{\partial}{\partial x}\right)^{k} u\right\rangle_{1-k, \mu, t}^{2}+\left\langle\Lambda_{x, s, \mu}^{-1 / 2}\left(\frac{\partial}{\partial y}\right)^{k} u\right\rangle_{1-k, \mu, t}^{2}\right. \\
& +\|\left\langle\Lambda_{x, y, \mu}^{-1 / 2}\left(\frac{\partial}{\partial z}\right)^{k} u \|_{1-k, \mu, t}^{2}\right\} \\
& \leqq C\left\{\|u(0)\|_{1, \mu}^{2}+\frac{1}{\mu}\|f\|_{0, \mu, t}^{2}+\frac{1}{\mu}\left\langle\Lambda_{y, z, \mu}^{1 / 2} g_{1}\right\rangle_{0, \mu, t}^{2}+\frac{1}{\mu}\left\langle\left\langle\Lambda_{x, z, \mu}^{1 / 2} g_{2}\right\rangle_{0, \mu, t}^{2}\right.\right. \\
& +\frac{1}{\mu}\left\langle\left\langle\left\langle\Lambda_{x, y, \mu}^{1 / 2} g_{3}\right\rangle\right\rangle_{0, \mu, t}^{2}\right\} \text {. }
\end{aligned}
$$


TheOREM 6. Let $\left(f, g_{1}, g_{2}, g_{3}, u_{0}, u_{1}\right)$ belongs to $C_{0}^{\infty}\left[\left(\overline{\boldsymbol{R}}_{+}^{1}\right)^{4}\right] \times\left[C_{0}^{\infty}\left[\left(\overline{\boldsymbol{R}}_{+}^{1}\right)^{8}\right]\right]^{5}$ and suppose that the conditions $\left(\mathrm{E}_{12}^{\prime \prime}\right),\left(\mathrm{C}_{35}\right),\left(\mathrm{II}_{1}\right),\left(\mathrm{II}_{3}\right),\left(\mathrm{II}_{6}\right),\left(\mathrm{III}_{1}\right),\left(\mathrm{III}_{3}\right)$ and (III $)_{5}$ hold.

Then, there exists a unique classical solution $u \in \mathscr{X}_{5, \mu}\left[\left(\boldsymbol{R}_{+}^{1}\right)^{4}\right]$ of the problem (III) which satisfies (2.4).

REMARK 3. We have Theorem 6 by the similar assumption in $(y, z)$ or $(x, z)$.

\section{§3. The root of the quadratic equation (2.1).} $b= \pm i$.

We are concerned with the root of the quadratic equation (2.1) where

Firstly, we treat the case where $b=i$. Then, the roots of (2.1) are $-i$ and $i((c-1) /(c+1))$. By (C.2) and the simple calculation, we have the following two cases for a root $i((c-1) /(c+1))$ :

(i)

$$
\operatorname{Re} c>0
$$

and

$$
\left|i\left(\frac{c-1}{c+1}\right)\right|<1 .
$$

(ii) $\quad c=i c_{1} \quad\left(c_{1}\right.$ is a positive number)

and

$$
\operatorname{Re}\left\{i\left(\frac{c-1}{c+1}\right)\right\}<0 .
$$

Secondly, we treat the case where $b=-i$. By the same arguments, we have the following two cases for a root $-i((c-1) /(c+1))$ :

$$
\operatorname{Re} c>0
$$

and

$$
\left|-i\left(\frac{c-1}{c+1}\right)\right|<1 \text {. }
$$

$$
c=i c_{2} \quad\left(c_{2}\right. \text { is a negative number) }
$$

and

$$
\operatorname{Re}\left\{-i\left(\frac{c-1}{c+1}\right)\right\}<0 .
$$

The above analysis is used to obtain the energy inequality in $\S 5$. 
§ 4. Mixed problem for symmetric hyperbolic system of first order. We consider the mixed problem

$$
\begin{aligned}
& \left\{\begin{array}{l}
\frac{\partial U}{\partial t}=A \frac{\partial U}{\partial x}+B \frac{\partial U}{\partial y}+K(t, x, y) U+F(t, x, y) \\
U(0, x, y)=U_{0}(x, y) \\
\left.P U\right|_{x=0}=G_{1}(t, y) \\
\left.Q U\right|_{y=0}=G_{2}(t, x)
\end{array}\right. \\
& (t, x, y) \in\left(R_{+}^{1}\right)^{s}
\end{aligned}
$$

where $U={ }^{t}\left(U_{1}, \cdots, U_{N}\right), A$ and $B$ are $N \times N$ constant Hermite matrices, $\operatorname{det}(A B) \neq 0, K, P$ and $Q$ are respectively $N \times N, p \times N$ and $q \times N$ smooth complex matrices, and are constant outside a compact set in $\left(\overline{\boldsymbol{R}}_{+}^{1}\right)^{8}, \overline{\boldsymbol{R}}_{+}^{1} \times \boldsymbol{R}^{1}$ and $\left(\overline{\boldsymbol{R}}_{+}^{1}\right)^{2}$.

We assume the following condition for the problem (4.1):

$$
\left\{\begin{array}{l}
((A U, U)) \geqq 0 \quad \text { for any } U \in \operatorname{Ker} P(t, y) \quad\left((t, y) \in \overline{\boldsymbol{R}}_{+}^{1} \times \boldsymbol{R}^{1}\right) \\
((B U, U)) \geqq C((U, U)) \quad \text { for any } U \in \operatorname{Ker} Q(t, x) \quad\left((t, x) \in\left(\overline{\boldsymbol{R}}_{+}^{1}\right)^{2}\right)
\end{array}\right.
$$

where $C$ is a positive constant.

We extend $K$ to the region $\{(t, x, y) \mid t \geqq 0, x \geqq 0, y<0\}$ as smooth functions and set $U(t, x, y)=0(y<0)$. Then, by the Fourier transform of (4.1) with respect to $y$, we have

$$
\left\{\begin{array}{l}
\hat{U}_{t}=A \hat{U}_{x}+i \eta B \hat{U}-B \cdot U(t, x, 0)+\widehat{K U}+\widehat{F} \\
\hat{U}(0, x, \eta)=\hat{U}_{0}(x, \eta) \\
\left.\widehat{P U}\right|_{x=0}=\hat{G}_{1}(t, \eta) \\
(t, x, \eta) \in\left(\boldsymbol{R}_{+}^{1}\right)^{2} \times R^{1}
\end{array}\right.
$$

where $\eta$ is the dual variable of $y$. We set

$$
e^{-\mu t} T_{\eta, \mu}^{-1 / 2} \hat{U}=W .
$$

Then, by (4.2) and (4.3), we obtain

$$
\begin{aligned}
W_{x}= & A^{-1} W_{t}+\mu A^{-1} W-e^{-\mu t} T_{\eta, \mu}^{-1 / 2}(i \eta) A^{-1} B \hat{U}+e^{-\mu t} A^{-1} B T_{\eta, \mu}^{-1 / 2} \cdot U(t, x, 0) \\
& -A^{-1} e^{-\mu t} T_{\eta, \mu}^{-1 / 2} \widehat{K U}-e^{-\mu t} A^{-1} T_{\eta, \mu}^{-1 / 2} \widehat{F}
\end{aligned}
$$

and $A^{-1}$ is a Hermite matrix. 


$$
\begin{aligned}
-\frac{d}{d x}[W, W]= & -\left[W_{x}, W\right]-\left[W, W_{x}\right] \\
= & -\left[A^{-1} W_{t}+\mu A^{-1} W-e^{-\mu t} T_{\eta, \mu}^{-1 / 2}(i \eta) A^{-1} B \hat{U}\right. \\
& +e^{-\mu t} A^{-1} B T_{\eta, \mu}^{-1 / 2} U(t, x, 0)-A^{-1} e^{-\mu t} T_{\eta, \mu}^{-1 / 2} \widehat{K U} \\
& \left.-e^{-\mu t} A^{-1} T_{\eta, \mu}^{-1 / 2} \hat{F}, W\right]-\left[W, A^{-1} W_{t}+\mu A^{-1} W\right. \\
& -e^{-\mu t} T_{\eta, \mu}^{-1 / 2}(i \eta) A^{-1} B \hat{U}+e^{-\mu t} A^{-1} B T_{\eta, \mu}^{-1 / 2} U(t, x, 0) \\
& \left.-e^{-\mu t} A^{-1} T_{\eta, \mu}^{-1 / 2} \widehat{K U}-e^{-\mu t} A^{-1} T_{\eta, \mu}^{-1 / 2} \widehat{F}\right] \\
= & -\frac{d}{d t}\left[A^{-1} W, W\right]-2 \mu\left[A^{-1} W, W\right] \\
& +\left[\left[e^{-\mu t} A^{-1} B T_{\eta, \mu}^{-1 / 2}(i \eta) \hat{U}, W\right]+\left[W, e^{-\mu t} A^{-1} B T_{\eta, \mu}^{-1 / 2}(i \eta) \hat{U}\right]\right\} \\
& -\left[A^{-1} B e^{-\mu t} T_{\eta, \mu}^{-1 / 2} U(t, x, 0), W\right]-\left[W, A^{-1} B e^{-\mu t} T_{\eta, \mu}^{-1 / 2} U(t, x, 0)\right] \\
& +\left[A^{-1} e^{-\mu t} T_{\eta, \mu}^{-1 / 2} \widehat{K U}, W\right]+\left[W, A^{-1} e^{-\mu t} T_{\eta, \mu}^{-1 / 2} \widehat{K U}\right] \\
& +\left[A^{-1} e^{-\mu t} T_{\eta, \mu}^{-1 / 2} \widehat{F}, W\right]+\left[W, A^{-1} e^{-\mu t} T_{\eta, \mu}^{-1 / 2} \hat{F}\right]
\end{aligned}
$$

Therefore, we have

$$
\begin{aligned}
\left\langle\Lambda_{y, \mu}^{-1 / 2} U\right\rangle_{0, \mu, t}^{2}= & \int_{0}^{t} \int_{-\infty}^{\infty} \int_{0}^{\infty}\left\{-\frac{d}{d x}((W, W))\right\} d x d \eta d t \\
\leqq & \frac{C_{1}}{\mu}\left\{\|U(t)\|\left\|_{0, \mu}^{2}+\right\| U(0)\|\|_{0, \mu}^{2}\right\}+C_{2}\|U\|_{0, \mu, t}^{2}+C_{8}\|U\|_{0, \mu, t}^{2} \\
& +\frac{C_{4}}{\mu}\langle U\rangle_{\rangle_{0, \mu, t}^{2}}^{2}+C_{8}\|U\|_{0, \mu, t}^{2}+\frac{C_{6}}{\mu}\|U\|_{0, \mu, t}^{2} \\
& +\frac{C_{7}}{\mu^{2}}\|F\|_{0, \mu, t}^{2}+C_{8}\|U\|_{0, \mu, t}^{2} .
\end{aligned}
$$

By (4.4), we obtain

LEMMA 4.1. Assume the condition (C.3). Let $U$ be the solution of the problem (4.1) which belongs to $\mathscr{H}_{1, \mu}\left[\left(\boldsymbol{R}_{+}^{1}\right)^{3}\right]$.

Then, there exist positive constants $C$ and $\mu_{0}$ such that

$$
\begin{aligned}
\left\langle\Lambda_{y, \mu}^{-1 / 2} U\right\rangle_{0, \mu, t}^{2} \leqq & C\left\{\frac{1}{\mu}\|U(t)\|\left\|_{0, \mu}^{2}+\frac{1}{\mu}\right\| U(0)\|\|_{0, \mu}^{2}+\frac{1}{\mu}\langle U U\rangle_{0, \mu, t}^{2}+\|U\|_{0, \mu, t}^{2}\right. \\
& \left.+\frac{1}{\mu^{2}}\|F\|_{0, \mu, t}^{2}\right\}
\end{aligned}
$$

for any $t \in \boldsymbol{R}_{+}^{1}$ and any $\mu \geqq \mu_{0}$.

TheOREM 4.2. Assume the condition (C.3). Let $U$ be the solution of the problem (4.1) which belongs to $\mathscr{H}_{1, \mu}\left[\left(\boldsymbol{R}_{+}^{1}\right)^{3}\right]$. 
Then, there exist positive constants $C$ and $\mu_{0}$ such that the energy inequality holds for any $t \in R_{+}^{1}$ and any $\mu \geqq \mu_{0}$

$$
\begin{aligned}
&\|U(t)\|_{0, \mu}^{2}+\mu\|U\|_{0, \mu, t}^{2}+\mu\left\langle\Lambda_{y, \mu}^{-1 / 2} U\right\rangle_{0, \mu, t}^{2}+\langle U\rangle_{0, \mu, t}^{2} \\
& \leqq C\left\{\|U(0)\|\left\|_{0, \mu}^{2}+\frac{1}{\mu}\right\| F \|_{0, \mu, t}^{2}+\frac{1}{\mu}\left\langle\Lambda_{y, \mu}^{1 / 2} G_{1}\right\rangle_{0, \mu, t}^{2}+\left\langle\left\langle G_{2}\right\rangle\right\rangle_{0, \mu, t}^{2}\right\} .
\end{aligned}
$$

Proof.

$$
\begin{aligned}
\frac{d}{d t}\left(e^{-\mu t} U(t), e^{-\mu t} U(t)\right) & \\
= & -2 \mu\left(e^{-\mu t} U, e^{-\mu t} U\right)+\left(e^{-\mu t}\left(A U_{x}+B U_{y}+K U+F\right), e^{-\mu t} U\right) \\
& +\left(e^{-\mu t} U, e^{-\mu t}\left(A U_{x}+B U_{y}+K U+F\right)\right) \\
\leqq & -C_{1} \mu\left(e^{-\mu t} U, e^{-\mu t} U\right)+\frac{C_{2}}{\mu}\left(e^{-\mu t} F, e^{-\mu t} F\right)-\left\langle A e^{-\mu t} U, e^{-\mu t} U\right\rangle \\
& -\left\langle B e^{-\mu t} U, e^{-\mu t} U\right\rangle
\end{aligned}
$$

where $C_{1}$ and $C_{2}$ are positive constants. By the condition (C.3), we obtain

$$
\begin{array}{r}
\left\langle A e^{-\mu t} U, e^{-\mu t} U\right\rangle \geqq-\delta \mu\left\langle\Lambda_{\nu, \mu}^{-1 / 2} e^{-\mu t} U, \Lambda_{\nu, \mu}^{-1 / 2} e^{-\mu t} U\right\rangle \\
-\frac{C_{8}}{\mu}\left\langle\Lambda_{\nu, \mu}^{1 / 2} e^{-\mu t} G_{1}, \Lambda_{\nu, \mu}^{1 / 2} e^{-\mu t} G_{1}\right\rangle
\end{array}
$$

and

$$
\left.\left.\left.《 B e^{-\mu t} U, e^{-\mu t} U\right\rangle \geqq C_{4} \| e^{-\mu t} U, e^{-\mu t} U\right\rangle-C_{b} \| e^{-\mu t} G_{2}, e^{-\mu t} G_{2}\right\rangle
$$

where $\delta$ is a sufficiently positive constants, $C_{3}, C_{4}$ and $C_{5}$ are positive constants. By (4.6), (4.7), (4.8) and (4.9), we get Theorem 4.2. Q.E.D.

\section{§5. Energy inequalities.}

Firstly, we transform the mixed problems (I), (II) and (III) for wave equation into the ones for symmetric hyperbolic system of first order.

We set respectively

$$
\left\{\begin{array} { l } 
{ z _ { 1 } = - i } \\
{ z _ { 2 } = i ( \frac { c - 1 } { c + 1 } ) }
\end{array} \text { or } \quad \left\{\begin{array}{l}
z_{1}=i \\
z_{2}=-i\left(\frac{c-1}{c+1}\right)
\end{array}\right.\right.
$$

for $b=i$ or $b=-i$, and use (5.1) for the problem (I).

LEmma 5.1. Assume the conditions (C.1) and (C.2). Then, the problem (I) is transformed into the following problem: 
where

$$
\left\{\begin{array}{l}
\frac{\partial U}{\partial t}=A_{1} \frac{\partial U}{\partial x}+B_{1} \frac{\partial U}{\partial y}+D_{1} U+F_{1}(t, x, y) \\
U(0, x, y)=U_{0}(x, y) \\
\left.P_{1} U\right|_{x=0}=G_{1}(t, y) \\
\left.Q_{1} U\right|_{y=0}=G_{2}(t, x) \\
(t, x, y) \in\left(R_{+}^{1}\right)^{3}
\end{array}\right.
$$

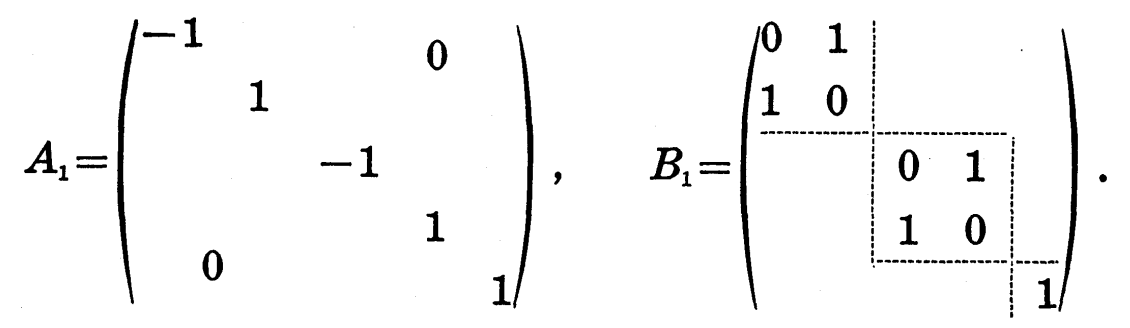

$D_{1}$ is a $5 \times 5$ constant matrix, $F_{1}=\left(f, z_{1} f, f, z_{2} f, 0\right)$

$$
\begin{aligned}
& P_{1}=Q_{1}=\left(\begin{array}{lllll}
1 & z_{2} & 0 & 0 & 0 \\
0 & 0 & 1 & z_{1} & 0
\end{array}\right) \\
& G_{1}=\left(-\frac{2}{c+1} g_{1},-\frac{2}{c+1} g_{1}\right), \quad G_{2}=\left(-\frac{2 b}{c+1} g_{2},-\frac{2 b}{c+1} g_{2}\right)
\end{aligned}
$$

and

$$
\begin{cases}\left(\left(A_{1} U, U\right)\right) \geqq 0 \text { for any } U \in \operatorname{Ker} P_{1} \\ \left(\left(B_{1} U, U\right)\right) \geqq 0 \text { for any } U \in \operatorname{Ker} Q_{1},\end{cases}
$$

Proof. We set

$$
U=\left(\begin{array}{c}
U_{1} \\
U_{2} \\
U_{8} \\
U_{4} \\
U_{5}
\end{array}\right)=\left(\begin{array}{c}
u_{t}-\left(u_{x}+\alpha u\right)+z_{1} u_{y} \\
z_{1}\left\{u_{t}+\left(u_{x}+\alpha u\right)\right\}+u_{y} \\
u_{t}-\left(u_{x}+\alpha u\right)+z_{2} u_{y} \\
z_{2}\left\{u_{t}+\left(u_{x}+\alpha u\right)\right\}+u_{y} \\
u
\end{array}\right)
$$

Then, by direct calculations, we have Lemma 5.1.

We treat the case where $\operatorname{Re} c>0$ in (I). We set

$$
V=\left(\begin{array}{l}
U_{1} \\
U_{2} \\
U_{\mathrm{b}}
\end{array}\right)
$$

for $U$ in (5.4). Then, by (3.1) and (3.3), we have 
LEMMA 5.2. The following fact holds:

$$
\left\{\begin{array}{l}
\frac{\partial V}{\partial t}=\left(\begin{array}{ccc}
-1 & 0 \\
& 1 & \\
0 & 1
\end{array}\right) \frac{\partial V}{\partial x}+\left(\begin{array}{lll}
0 & 1 & 0 \\
1 & 0 & 0 \\
0 & 0 & 1
\end{array}\right) \frac{\partial V}{\partial y}+D_{11} V+E_{11} W+H_{1} \\
\quad=A_{11} V_{x}+B_{11} V_{y}+D_{11} V+E_{11} W+H_{1} \\
V(0, x, y)=V_{0}(x, y) \\
\left.P_{11} V\right|_{x=0}=-\frac{2}{c+1} g_{1} \\
\left.Q_{11} V\right|_{y=0}=-\frac{2 b}{c+1} g_{2} \\
(t, x, y) \in\left(R_{+}^{1}\right)^{3}
\end{array}\right.
$$

where $D_{11}$ and $E_{11}$ are respectively $3 \times 3$ and $3 \times 2$ constant matrices, $W=$ ${ }^{t}\left(U_{3}, U_{4}\right), H_{1}={ }^{t}\left(f, z_{1} f, 0\right), P_{11}=Q_{11}=\left(1, z_{2}, 0\right)$ and for a positive constant $C$

$$
\begin{cases}\left(\left(A_{11} V, V\right)\right) \geqq C((V, V)) & \text { for any } V \in \operatorname{Ker} P_{11} \\ \left(\left(B_{11} V, V\right)\right) \geqq 0 & \text { for any } V \in \operatorname{Ker} Q_{11} .\end{cases}
$$

Next, we treat the case where $\operatorname{Re} c=0$ in (I). We set

$$
V=\left(\begin{array}{l}
U_{1} \\
U_{2} \\
U_{5}
\end{array}\right)
$$

for $U$ in (5.4). Then, by (3.2) and (3.4), we have

LEMMA 5.3. The following fact holds:

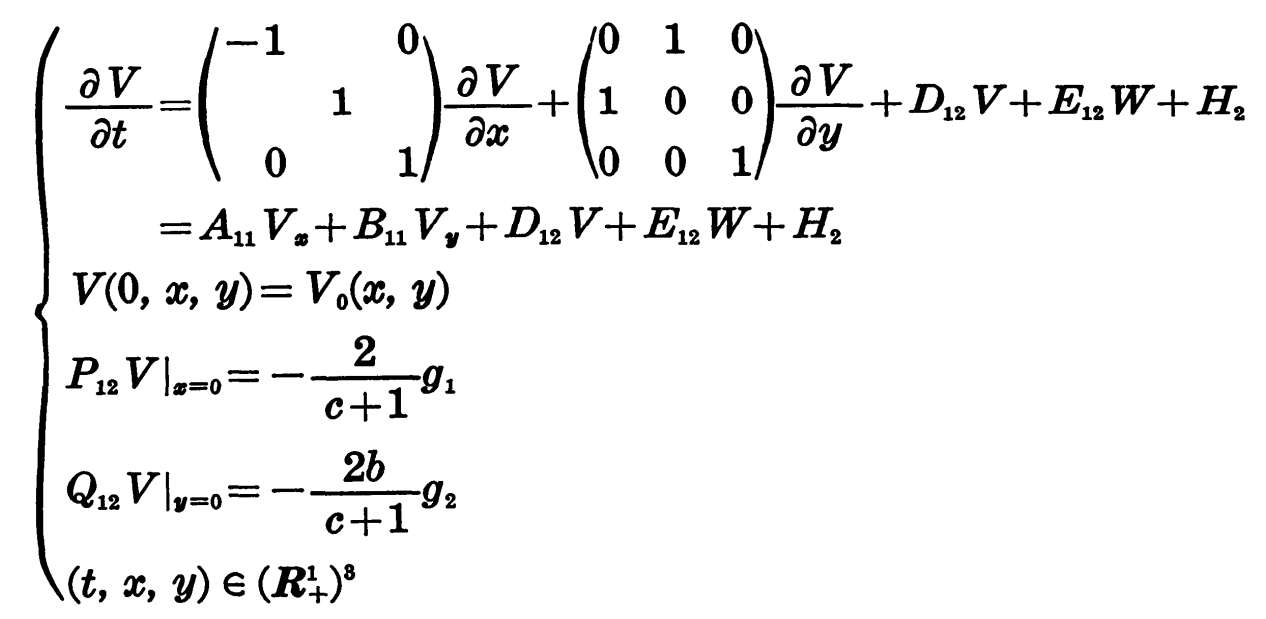

where $D_{12}$ and $E_{12}$ are respectively $3 \times 3$ and $3 \times 2$ constant matrices, $W=$ 
${ }^{t}\left(U_{3}, U_{4}\right), H_{2}=\left(f, z_{1} f, 0\right), P_{12}=Q_{12}=\left(1, z_{2}, 0\right)$ and for a posiitive constant $C$

$$
\begin{cases}\left(\left(A_{11} V, V\right)\right) \geqq 0 & \text { for any } V \in \operatorname{Ker} P_{12} \\ \left(\left(B_{11} V, V\right)\right) \geqq C((V, V)) & \text { for any } V \in \operatorname{Ker} Q_{12} .\end{cases}
$$

Now, we consider the problems (II) and (III).

LEMMA 5.4. The problem (II) is transformed into the following problem:

$$
\left\{\begin{array}{l}
\frac{\partial U}{\partial t}=A_{2} \frac{\partial U}{\partial x}+B_{2} \frac{\partial U}{\partial y}+D_{2} U+F_{2} \\
U(0, x, y)=U_{0}(x, y) \\
\left.P_{2} U\right|_{x=0}=-2 g_{1} \\
\left.Q_{2} U\right|_{y=0}=\sqrt{2} g_{2} \\
(t, x, y) \in\left(R_{+}^{1}\right)^{3}
\end{array}\right.
$$

where

$$
A_{2}=\left(\begin{array}{ccc}
-1 & & 0 \\
& 1 & 0 \\
0 & & 0
\end{array}\right), \quad B_{2}=\left(\begin{array}{cccc}
0 & 0 & \frac{1}{\sqrt{2}} & 0 \\
0 & 0 & \frac{1}{\sqrt{2}} & 0 \\
\frac{1}{\sqrt{2}} & \frac{1}{\sqrt{2}} & 0 & 0 \\
0 & 0 & 0 & 0
\end{array}\right)
$$

$D_{2}$ is a $4 \times 4$ constant matrix, $F_{2}={ }^{t}(f, f, 0,0)$

$$
P_{2}=(1,-1,0,0), \quad Q_{2}=(0,0,1,0)
$$

and

$$
\left\{\begin{array}{l}
\left(\left(A_{2} U, U\right)\right) \geqq 0 \text { for any } U \in \operatorname{Ker} P_{2} \\
\left(\left(B_{2} U, U\right)\right) \geqq 0 \text { for any } U \in \operatorname{Ker} Q_{2} .
\end{array}\right.
$$

Proof. We set

$$
U=\left(\begin{array}{c}
U_{1} \\
U_{2} \\
U_{8} \\
U_{\downarrow}
\end{array}\right)=\left(\begin{array}{c}
u_{t}-\left(u_{x}+\alpha u\right) \\
u_{t}+\left(u_{x}+\alpha u\right) \\
\sqrt{2}\left(u_{y}+\beta u\right) \\
u
\end{array}\right) .
$$

Then, by direct calculations, we have Lemma 5.4. 
LEMMA 5.5. The problem (III) is transformed into the following problem:

where

$$
\left\{\begin{array}{l}
\frac{\partial U}{\partial t}=A_{8} \frac{\partial U}{\partial x}+B_{8} \frac{\partial U}{\partial y}+E_{3} \frac{\partial U}{\partial z}+D_{8} U+F_{8} \\
U(0, x, y, z)=U_{0}(x, y, z) \\
\left.P_{8} U\right|_{z=0}=G_{1} \\
\left.Q_{8} U\right|_{y=0}=G_{2} \\
\left.R_{8} U\right|_{z=0}=G_{8} \\
(t, x, y, z) \in\left(R_{+}^{1}\right)^{4}
\end{array}\right.
$$

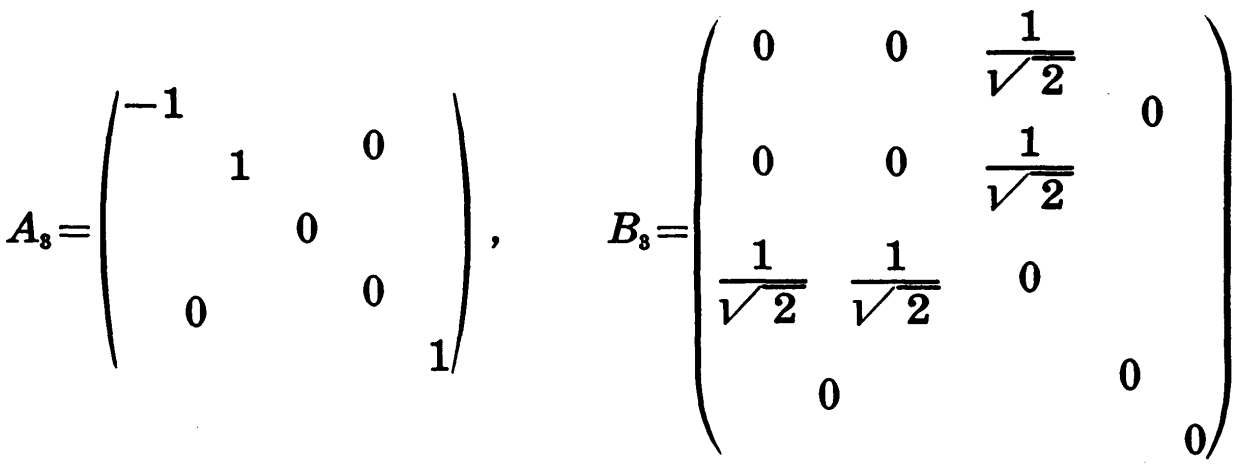

$$
\begin{aligned}
& E_{8}=\left(\begin{array}{ccccc}
0 & 0 & 0 & \frac{1}{\sqrt{2}} & 0 \\
0 & 0 & 0 & \frac{1}{\sqrt{2}} & 0 \\
0 & 0 & 0 & 0 & 0 \\
\frac{1}{\sqrt{2}} & \frac{1}{\sqrt{2}} & 0 & 0 & 0 \\
0 & 0 & 0 & 0 & 0
\end{array}\right) \\
& F={ }^{t}(f, f, 0,0,0), \quad P_{8}=(-1,1,0,0,0) \\
& Q_{8}=(0,0,1,0,0), \quad R_{8}=(0,0,0,1,0) \\
& G_{1}=-2 g_{1}, \quad G_{2}=\sqrt{2} g_{2}, \quad G_{8}=\sqrt{2} g_{3}
\end{aligned}
$$

and

$$
\left\{\begin{array}{l}
\left(\left(A_{8} U, U\right)\right) \geqq 0 \text { for any } U \in \operatorname{Ker} P_{8} \\
\left(\left(B_{8} U, U\right)\right) \geqq 0 \text { for any } U \in \operatorname{Ker} Q_{8} \\
\left(\left(E_{8} U, U\right)\right) \geqq 0 \text { for any } U \in \operatorname{Ker} R_{8} .
\end{array}\right.
$$

Proof. We set 


$$
U=\left(\begin{array}{c}
U_{1} \\
U_{2} \\
U_{3} \\
U_{4} \\
U_{8}
\end{array}\right)=\left(\begin{array}{c}
u_{t}-\left(u_{x}+\alpha u\right) \\
u_{t}+\left(u_{x}+\alpha u\right) \\
\sqrt{2}\left(u_{y}+\beta u\right) \\
\sqrt{2}\left(u_{z}+\gamma u\right) \\
u
\end{array}\right) .
$$

Then, by direct calculations, we have Lemma 5.5.

Secondly, we shall get the energy inequalities for the problems (I), (II) and (III).

LEMma 5.6. (1) Let $u$ belong to $\mathscr{H}_{2, \mu}\left[\left(\boldsymbol{R}_{+}^{1}\right)^{3}\right]$. Then, we have

$$
\left\{\begin{array}{l}
\text { (i) } \int_{0}^{\infty} e^{-2 \mu t}\left|\left(\Lambda_{y, \mu}^{-1 / 2} u_{y}\right)(t, 0, y)\right|^{2} d y \leqq C|||u(t)| \|_{1, \mu}^{2} \\
\text { (ii) } \int_{0}^{\infty} e^{-2 \mu t}\left|\left(\Lambda_{x, \mu}^{-1 / 2} u_{x}\right)(t, x, 0)\right|^{2} d x \leqq C|| \mid u(t)\|\|_{1, \mu}^{2}
\end{array}\right.
$$

where $C$ is a positive constant, any $t \in R_{+}^{1}$ and any $\mu \geqq \mu_{0}\left(\mu_{0}\right.$ is a positive constant).

(2) Let $u$ belong to $\mathscr{H}_{2, \mu}\left[\left(\boldsymbol{R}_{+}^{1}\right)^{4}\right]$. Then, we have

$$
\left\{\begin{array}{c}
\text { ( i ) } \int_{0}^{\infty} \int_{0}^{\infty} e^{-2 \mu t}\left\{\left|\left(\Lambda_{y, z, \mu}^{-1 / 2} u_{y}\right)(t, 0, y, z)\right|^{2}+\left|\left(\Lambda_{y, z, \mu}^{-1 / 2} u_{z}\right)(t, 0, y, z)\right|^{2}\right\} d y d z \\
\text { (ii) } \int_{0}^{\infty} \int_{0}^{\infty} e^{-2 \mu t}\left\{\left|\left(\Lambda_{x, z, \mu}^{-1 / 2} u_{x}\right)(t, x, 0, z)\right|^{2}+\left|\left(\Lambda_{x, z, \mu}^{-1 / 2} u_{z}\right)(t, x, 0, z)\right|^{2}\right\} d x d z \\
\leqq C\left|\|u(t) \mid\|_{1, \mu}^{2}\right. \\
\text { (iii) } \int_{0}^{\infty} \int_{0}^{\infty} e^{-2 \mu t}\left\{\left|\left(\Lambda_{x, y, \mu}^{-1 / 2} u_{x}\right)(t, x, y, 0)\right|^{2}+\left|\left(\Lambda_{x, y, \mu}^{-1 / 2} u_{y}\right)(t, x, y, 0)\right|^{2}\right\} d x d y \\
\leqq C\left|\|u(t) \mid\|_{1, \mu}^{2}\right.
\end{array}\right.
$$

where $C$ is a positive constant, any $t \in \boldsymbol{R}_{+}^{1}$ and any $\mu \geqq \mu_{0}\left(\mu_{0}\right.$ is a positive constant).

The proof of this lemma is not given here, because it is popular.

Lemma 5.7. (1) Let $u$ belong to $\mathscr{H}_{2, \mu}\left[\left(\boldsymbol{R}_{+}^{1}\right)^{3}\right]$. Then, there exist positive constants $C$ and $\mu_{0}$ such that for any $t \in \boldsymbol{R}_{+}^{1}$ and any $\mu \geqq \mu_{0}$

$$
\begin{aligned}
& \|\| \mu u(t)\left\|_{0, \mu}^{2}+\mu \mid\right\| \mu u \|_{0, \mu, t}^{2}+\mu\left\langle\Lambda_{y, \mu}^{-1 / 2} \mu u\right\rangle_{0, \mu, t}^{2}+\mu\left\langle\left\langle\Lambda_{x, \mu}^{-1 / 2} \mu u\right\rangle_{0, \mu, t}^{2}\right. \\
& \leqq C\left\{\|u(0)\|\left\|_{1, \mu}^{2}+\mu\right\| u_{t}\left\|_{0, \mu, t}^{2}+\mu\right\| u_{x}\left\|_{0, \mu, t}^{2}+\mu\right\| u_{y} \|_{0, \mu, t}^{2}\right\} \text {. }
\end{aligned}
$$

(2) Let $u$ belong to $\mathscr{H}_{2, \mu}\left[\left(\boldsymbol{R}_{+}^{1}\right)^{4}\right]$. Then, there exist positive constants 
$C$ and $\mu_{0}$ such that for any $t \in \boldsymbol{R}_{+}^{1}$ and any $\mu \geqq \mu_{0}$

$$
\begin{aligned}
& \|\| \mu u(t)\left\|_{0, \mu}^{2}+\mu\right\| \mu \mu u \|_{0, \mu, t}^{2}+\mu\left\langle\Lambda_{\nu, z, \mu}^{-1 / 2} \mu u\right\rangle_{0, \mu, t}^{2} \\
& +\mu\left\langle\left\langle\Lambda_{x, z, \mu}^{-1 / 2} \mu u\right\rangle_{0, \mu, t}^{2}+\mu\left\langle\left\langle\left\langle\Lambda_{x, \nu}^{-1 / 2} \mu u\right\rangle\right\rangle_{0, \mu, t}^{2}\right.\right. \\
& \leqq C\left\{\|u(0)\|_{1, \mu}^{2}+\mu\left\|u_{t}\right\|_{0, \mu, t}^{2}+\mu\|\| u_{x}\left\|_{0, \mu, t}^{2}+\mu\right\| u_{y}\left\|_{0, \mu, t}^{2}+\mu\right\|\left\|u_{s}\right\|_{0, \mu, t}^{2}\right\} \text {. }
\end{aligned}
$$

Proof. It follows easily

$$
\begin{aligned}
\frac{d}{d t}\left(e^{-\mu t} u(t), e^{-\mu t} u(t)\right) & =-2 \mu\left(e^{-\mu t} u, e^{-\mu t} u\right)+2 \operatorname{Re}\left(e^{-\mu t} u_{t}, e^{-\mu t} u\right) \\
& \leqq-C_{1} \mu\left(e^{-\mu t} u, e^{-\mu t} u\right)+\frac{C_{2}}{\mu}\left(e^{-\mu t} u_{t}, e^{-\mu t} u_{t}\right)
\end{aligned}
$$

where $C_{1}$ and $C_{2}$ are positive constants. Then, we get

$$
\|\| \mu u(t)\left\|_{0, \mu}^{2}+C_{1} \mu\right\| \mu u\left\|_{0, \mu}^{2} \leqq\right\| u(0) \mid\left\|_{1, \mu}^{2}+C_{2} \mu\right\| u_{t} \|_{0, \mu, t}^{2} .
$$

Also, we obtain

$$
\begin{aligned}
\langle\mu u\rangle_{0, \mu, t}^{2} & =\int_{0}^{t}\left\langle e^{-\mu t} \mu u, e^{-\mu t} \mu u\right\rangle d t \\
& =-\mu \int_{0}^{t}\left\{\left(e^{-\mu t} u_{x}, e^{-\mu t} \mu u\right)+\left(e^{-\mu t} \mu u, e^{-\mu t} u_{x}\right)\right\} d t \\
& \leqq C \cdot \mu\left(\|\mu u\|_{0, \mu, t}^{2}+\left\|u_{x}\right\|_{0, \mu, t}^{2}\right)
\end{aligned}
$$

and similarly, we have

$$
\langle\mu u\rangle_{0, \mu, t}^{2} \leqq C \cdot \mu\left(\|\mu u\|_{0, \mu, t}^{2}+\left\|u_{y}\right\|_{0, \mu, t}^{2}\right)
$$

where $C$ is a positive constant. Also, we have

$$
\left\{\begin{array}{l}
\langle\mu u\rangle_{0, \mu, t}^{2} \geqq \mu\left\langle\Lambda_{y, \mu}^{-1 / 2} \mu u\right\rangle_{0, \mu, t}^{2} \\
\langle\mu u\rangle_{0, \mu, t}^{2} \geqq \mu\left\langle\left\langle\Lambda_{x, \mu}^{-1 / 2} \mu u\right\rangle_{0, \mu, t}^{2}\right.
\end{array}\right.
$$

By (5.21), (5.22), (5.23) and (5.24), we have (5.19). By the same method, we have (5.20).

Q.E.D.

Proof of Theorem 1. We treat the case where Re $c>0$ in (I) because the similar method and Lemma 5.3 are applied to the case where $\operatorname{Re} c=0$ in (I).

By results in $\S 4$, Lemma 5.2 and Lemma 5.6, we have

$$
\mu \sum_{k=0}^{1}\left\{\left\langle\Lambda_{y, \mu}^{-1 / 2}\left(\frac{\partial}{\partial x}\right)^{k} u\right\rangle_{1-k, \mu, t}^{2}+\left\langle\Lambda_{x, \mu}^{-1 / 2}\left(\frac{\partial}{\partial y}\right)^{k} u\right\rangle_{1-k, \mu, t}^{2}\right\}
$$




$$
\begin{aligned}
\leqq & C\left\{\|u(0)\|\left\|_{1, \mu}^{2}+\frac{1}{\mu}\right\| f \|_{0, \mu, t}^{2}+\left\langle g_{1}\right\rangle_{0, \mu, t}^{2}+\frac{1}{\mu}\left\langle\left\langle\Lambda_{x, \mu}^{1 / 2} g_{2}\right\rangle_{0, \mu, t}^{2}\right.\right. \\
& \left.+\mu\|u\|_{1, \mu, t}^{2}+\frac{1}{\mu}\left\|U_{3}\right\|_{0, \mu, t}^{2}+\frac{1}{\mu}\left\|U_{4}\right\|_{0, \mu, t}^{2}\right\}
\end{aligned}
$$

where $U_{3}$ and $U_{4}$ in (5.4). For $U$ in (5.4), we obtain

$$
\begin{aligned}
& \frac{d}{d t}\left(e^{-\mu t} U, e^{-\mu t} U\right) \\
&=-2 \mu\left(e^{-\mu t} U, e^{-\mu t} U\right)+\left(e^{-\mu t} U_{t}, e^{-\mu t} U\right)+\left(e^{-\mu t} U, e^{-\mu t} U_{t}\right) \\
&=-2 \mu\left(e^{-\mu t} U, e^{-\mu t} U\right)+\left(e^{-\mu t}\left(A_{1} U_{x}+B_{1} U_{y}+D_{1} U+F_{1}\right), e^{-\mu t} U\right) \\
&+\left(e^{-\mu t} U, e^{-\mu t}\left(A_{1} U_{x}+B_{1} U_{y}+D_{1} U+F_{1}\right)\right) \\
& \leqq-C_{1} \mu\left(e^{-\mu t} U, e^{-\mu t} U\right)+\frac{C_{2}}{\mu}\left(e^{-\mu t} F_{1}, e^{-\mu t} F_{1}\right) \\
&-\left\langle A_{1} e^{-\mu t} U, e^{-\mu t} U\right\rangle-\left\langle\left\langle B_{1} e^{-\mu t} U, e^{-\mu t} U\right\rangle\right.
\end{aligned}
$$

and by (5.3), we have

$$
\left\{\begin{aligned}
\left\langle A_{1} e^{-\mu t} U, e^{-\mu t} U\right\rangle \geqq & -\delta \mu\left\langle\Lambda_{y, \mu}^{-1 / 2} e^{-\mu t} U, \Lambda_{y, \mu}^{-1 / 2} e^{-\mu t} U\right\rangle \\
& -\frac{C_{3}}{\mu}\left\langle\Lambda_{y, \mu}^{1 / 2} e^{-\mu t} G_{1}, \Lambda_{y, \mu}^{1 / 2} e^{-\mu t} G_{1}\right\rangle \\
\left\langle B_{1} e^{-\mu t} U, e^{-\mu t} U\right\rangle \geqq & -\delta \mu\left\langle\left\langle\Lambda_{x, \mu}^{-1 / 2} e^{-\mu t} U, \Lambda_{x, \mu}^{-1 / 2} e^{-\mu t} U\right\rangle\right. \\
& -\frac{C_{4}}{\mu}\left\langle\left\langle\Lambda_{x, \mu}^{1 / 2} e^{-\mu t} G_{2}, \Lambda_{x, \mu}^{1 / 2} e^{-\mu t} G_{2}\right\rangle\right\rangle
\end{aligned}\right.
$$

where $\delta$ is a sufficiently small positive constant. By (5.4), (5.25), (5.26) and (5.27), we obtain Theorem 1.

Q.E.D.

Proof of Theorem 3. For $U$ in (5.13), we set $U(t, x, y)=0(y<0)$. By the Fourier transform of (5.11) with respect to $y$, we have

$$
\left\{\begin{array}{l}
\hat{U}_{t}=A_{2} \hat{U}_{x}+i \eta B_{2} \hat{U}-B_{2} \cdot U(t, x, 0)+D_{2} \hat{U}+\hat{F}_{2} \\
\hat{U}(0, x, \eta)=\hat{U}_{0}(x, \eta) \\
\left.P \hat{U}\right|_{x=0}=\hat{G}_{1} \\
(t, x, \eta) \in\left(\boldsymbol{R}_{+}^{1}\right)^{2} \times \boldsymbol{R}^{1}
\end{array}\right.
$$

where $\hat{U}=\int_{-\infty}^{\infty} e^{-i y \cdot \eta} U(t, x, y) d y$. We set

$$
\left\{\begin{array}{l}
e^{-\mu t} T_{\eta, \mu}^{-1} \hat{U}=V \\
e^{-\mu t} T_{\eta, \mu}^{-1 / 2} \hat{U}=W .
\end{array}\right.
$$


By $\left.Q_{2} U\right|_{y=0}=\sqrt{2} g_{2},(5.28)$ and (5.29), we obtain

$$
V_{t}=A_{2} V_{z}+e^{-\mu t} T_{\eta, \mu}^{-1}(i \eta) B_{2} \hat{U}-e^{-\mu t} T_{\eta, \mu}^{-1} B_{2} \cdot U(t, x, 0)-\mu V+D_{2} V+e^{-\mu t} T_{\eta, \mu}^{-1} \hat{F}_{2}
$$

and

$$
B_{2} \cdot U(t, x, 0)=\left(g_{2}, g_{2}, \frac{U_{1}+U_{2}}{\sqrt{2}}, 0\right)
$$

Therefore, we obtain

$$
\begin{aligned}
-A_{4} V_{x}= & -A_{2} V_{t}+A_{2} B_{2} e^{-\mu t} T_{\eta, \mu}^{-1}(i \eta) \hat{U}-e^{-\mu t} T_{\eta, \mu}^{-1} \cdot H \\
& +A_{2}\left(-\mu V+D_{2} V+e^{-\mu t} T_{\eta_{, \mu}}^{-1} \hat{F}_{2}\right)
\end{aligned}
$$

where

$$
A_{4}=\left(\begin{array}{llll}
1 & & & 0 \\
& 1 & & \\
& 0 & 0 & \\
& & & 1
\end{array}\right)
$$

and

$$
H={ }^{t}\left(-g_{2}, g_{2}, 0,0\right) \text {. }
$$

By $\left(V, e^{-\mu t} \hat{U}\right)=(W, W)$ and (5.31), we get

$$
\begin{aligned}
& -\frac{d}{d x}\left[A_{4} W, W\right] \\
& =-\left[A_{4} W_{z}, W\right]-\left[W, A_{4} W_{z}\right] \\
& =-\left[A_{4} V_{s}, e^{-\mu t} \hat{U}\right]-\left[e^{-\mu t} \hat{U}, A_{4} V_{x}\right] \\
& =\left[-A_{2} V_{t}+A_{2} e^{-\mu t} T_{\eta_{, \mu}}^{-1}(i \eta) B_{2} \hat{U}-e^{-\mu t} T_{\eta_{, \mu}}^{-1} \cdot H\right. \\
& \left.+A_{2}\left(-\mu V+D_{2} V+e^{-\mu t} T_{\eta, \mu}^{-1} \hat{F}_{2}\right), e^{-\mu t} \hat{U}\right] \\
& +\left[e^{-\mu t} \hat{U},-A_{2} V_{t}+A_{2} e^{-\mu t} T_{\eta, \mu}^{-1}(i \eta) B_{2} \hat{U}-e^{-\mu t} T_{\eta, \mu}^{-1} \cdot H\right. \\
& \left.+A_{2}\left(-\mu V+D_{2} V+e^{-\mu t} T_{\eta, \mu}^{-1} \hat{F}_{2}\right)\right] \\
& =-\frac{d}{d t}\left[A_{2} W, W\right]+\left[A_{2} B_{2} e^{-\mu t} T_{\eta, \mu}^{-1}(i \eta) \hat{U}, e^{-\mu t} \hat{U}\right] \\
& +\left[e^{-\mu t} \hat{U}, A_{2} B_{2} e^{-\mu t} T_{\eta, \mu}^{-1}(i \eta) \hat{U}\right]-\left[e^{-\mu t} T_{\eta, \mu}^{-1} \cdot H, e^{-\mu t} \hat{U}\right] \\
& -\left[e^{-\mu t} \hat{U}, e^{-\mu t} T_{\eta, \mu}^{-1} \cdot H\right]+\left[A_{2}\left(-\mu V+D_{2} V+e^{-\mu t} T_{\eta, \mu}^{-1} \hat{F}_{2}\right), e^{-\mu t} \hat{U}\right] \\
& +\left[e^{-\mu t} \hat{U}, A_{2}\left(-\mu V+D_{2} V+e^{-\mu t} T_{\eta, \mu}^{-1} \hat{F^{\prime}}\right)\right] \text {. }
\end{aligned}
$$

By Lemma 5.6 and (5.33), we have 


$$
\begin{aligned}
\left\langle\Lambda_{y, \mu}^{-1 / 2} U\right\rangle_{0, \mu, t}^{2} \leqq & \frac{C_{1}}{\mu}\left(\left.\|U(t)\|\right|_{0, \mu} ^{2}+\left.\|U(0)\|\right|_{0, \mu} ^{2}\right)+C_{2}\|U\|_{0, \mu, t}^{2} \\
& +\frac{C_{3}}{\mu^{2}}\left\|F_{2}\right\|_{0, \mu, t}^{2}+\frac{C_{4}}{\mu^{2}}\left\langle\Lambda_{x, \mu}^{1 / 2} G_{2}\right\rangle_{0, \mu, t}^{2}
\end{aligned}
$$

where $C_{1}, C_{2}, C_{3}$ and $C_{4}$ are positive constants. By the symmetricity of the condition in $x$ and $y$, we get

$$
\begin{aligned}
\left\langle\Lambda_{x, \mu}^{-1 / 2} U\right\rangle_{0, \mu, t}^{2} \leqq & \frac{C_{1}^{\prime}}{\mu}\left(\left.\|U(t)\|\right|_{0, \mu} ^{2}+\| U(0)||_{0, \mu}^{2}\right)+C_{2}^{\prime}\|U\|_{0, \mu, t}^{2} \\
& +\frac{C_{8}}{\mu^{2}}\left\|F_{2}\right\|_{0, \mu, t}^{2}+\frac{C_{4}^{\prime}}{\mu^{2}}\left\langle\Lambda_{y, \mu}^{1 / 2} G_{1}\right\rangle_{0, \mu, t}^{2}
\end{aligned}
$$

where $C_{1}^{\prime}, C_{2}^{\prime}, C_{3}^{\prime}$ and $C_{4}^{\prime}$ are positive constants. For $U$ in (5.13), we obtain

$$
\begin{aligned}
\frac{d}{d t}\left(e^{-\mu t} U, e^{-\mu t} U\right) \leqq & -C_{1} \mu\left(e^{-\mu t} U, e^{-\mu t} U\right)+\frac{C_{2}}{\mu}\left(e^{-\mu t} F_{2}, e^{-\mu t} F_{2}\right) \\
& -\left\langle A_{2} e^{-\mu t} U, e^{-\mu t} U\right\rangle-\left\langle\left\langle B_{2} e^{-\mu t} U, e^{-\mu t} U\right\rangle\right\rangle
\end{aligned}
$$

and by (5.12), we have

$$
\left\{\begin{aligned}
\left\langle A_{2} e^{-\mu t} U, e^{-\mu t} U\right\rangle \geqq & -\delta u\left\langle\Lambda_{y, \mu}^{-1 / 2} e^{-\mu t} U, \Lambda_{y, \mu}^{-1 / 2} e^{-\mu t} U\right\rangle \\
& -C_{3}\left\langle\Lambda_{y, \mu}^{1 / 2} e^{-\mu t} G_{1}, \Lambda_{y, \mu}^{1 / 2} e^{-\mu t} G_{1}\right\rangle \\
\left\langle B_{2} e^{-\mu t} U, e^{-\mu t} U\right\rangle \geqq & -\delta \mu\left\langle\left\langle\Lambda_{x, \mu}^{-1 / 2} e^{-\mu t} U, \Lambda_{x, \mu}^{-1 / 2} e^{-\mu t} U\right\rangle\right. \\
& -C_{4}\left\langle\left\langle\Lambda_{x, \mu}^{1 / 2} e^{-\mu t} G_{2}, \Lambda_{x, \mu}^{1 / 2} e^{-\mu t} G_{2}\right\rangle\right\rangle
\end{aligned}\right.
$$

where $\delta$ is a sufficiently small positive constant, $C_{1}, C_{2}, C_{3}$ and $C_{4}$ are positive constants. By (5.34), (5.35), (5.36) and (5.37), we get Theorem 3. Q.E.D.

Proof of Theorem 5. For $U$ in (5.16), we set $U(t, x, y, z)=0(y<0$ or $z<0)$. By the Fourier transform of (5.14) with respect to $(y, z)$, we have

$$
\hat{U}_{t}=A_{8} \hat{U}_{x}+i \eta B_{3} \hat{U}+i \zeta E_{3} \hat{U}-B_{3} \tilde{U}(t, x, 0, \zeta)-E_{8} \tilde{U}(t, x, \eta, 0)+D_{3} \hat{U}+\hat{F}_{8}
$$

where

$$
\left\{\begin{array}{l}
\hat{U}=\int_{-\infty}^{\infty} \int_{-\infty}^{\infty} e^{-i(y \cdot \eta+z \cdot \zeta)} U(t, x, y, z) d y d z \\
\widetilde{U}=\int_{-\infty}^{\infty} e^{-i z \cdot \zeta} U(t, x, 0, z) d z \\
\tilde{U}=\int_{-\infty}^{\infty} e^{-i y \cdot \eta} U(t, x, y, 0) d y
\end{array}\right.
$$

We set 


$$
\left\{\begin{array}{l}
e^{-\mu t} T_{\eta, \zeta, \mu}^{-1} \hat{U}=V \\
e^{-\mu t} T_{\eta, \zeta, \mu}^{-1 / 2} \hat{U}=W .
\end{array}\right.
$$

By $\left.Q_{3} U\right|_{y=0}=G_{2},\left.R_{8} U\right|_{s=0}=G_{3}$, (5.38) and (5.39), we obtain

and

$$
\begin{aligned}
V_{t}= & A_{8} V_{x}+e^{-\mu t} T_{\eta, \zeta, \mu}^{-1}\left\{(i \eta) B_{8} \hat{U}+(i \zeta) E_{8} \hat{U}\right\} \\
& -e^{-\mu t} T_{\eta, \zeta, \mu}^{-1}\left\{B_{2} \widetilde{U}(t, x, 0, \zeta)+E_{8} \tilde{U}(t, x, \eta, 0)\right\} \\
& -\mu V+D_{3} V+e^{-\mu t} T_{\eta, \zeta, \mu}^{-1} \hat{F}_{3}
\end{aligned}
$$

\section{d}

Therefore, we have

$$
\begin{aligned}
-A_{5} V_{x}= & A_{8} V_{t}-A_{8}\left[e^{-\mu t} T_{\eta, \zeta, \mu}^{-1}\left(i \eta B_{3} \hat{U}+i \zeta E_{8} \hat{U}\right)\right] \\
& -e^{\mu t} T_{\eta, \zeta, \mu}^{-1}\left(H_{1} \widetilde{U}+H_{2} \tilde{U}\right)+A_{8}\left[-\mu V+D_{8} V+e^{-\mu t} T_{\eta, \zeta,}^{-1} \hat{F}_{8}\right]
\end{aligned}
$$

where

$$
A_{5}=\left(\begin{array}{lllll}
1 & & & & \\
& 1 & & 0 & \\
& & 0 & & \\
0 & & 0 & \\
& & & & 1
\end{array}\right)
$$

and

$$
\left\{\begin{array}{l}
H_{1}={ }^{t}\left(-\frac{\widetilde{G}_{2}}{\sqrt{2}}, \frac{\widetilde{G}_{2}}{\sqrt{2}}, 0,0,0\right) \\
H_{2}={ }^{t}\left(-\frac{\tilde{G}_{3}}{\sqrt{2}}, \frac{\widetilde{G}_{3}}{\sqrt{2}}, 0,0,0\right) .
\end{array}\right.
$$

By the same arguments as the one for the proof of Theorem 3, Lemma 5.5, Lemma 5.6 and (5.42), we have Theorem 5.

§ 6. The existence of the solution (I).

In this section, we shall prove Theorems 2 and 4.

LEMMA 6.1. Let $u$ be the solution of the problem (I) which belongs to $\mathscr{H}_{6, \mu}\left[\left(\boldsymbol{R}_{+}^{1}\right)^{8}\right]$. 
Then, there exist positive constants $C$ and $\mu_{0}$ such that the following inequality holds for any $t \in \boldsymbol{R}_{+}^{1}$ and any $\mu \geqq \mu_{0}$

$$
\begin{array}{r}
\|u(t)\|\left\|_{\delta, \mu}^{2}+\mu\right\|\|u\|_{\delta, \mu, t}^{2}+\mu \sum_{k=0}^{1}\left\{\left\langle\Lambda_{y, \mu}^{-1 / 2}\left(\frac{\partial}{\partial x}\right)^{k} u\right\rangle_{s-k, \mu, t}^{2}+\left\langle\Lambda_{x, \mu}^{-1 / 2}\left(\frac{\partial}{\partial y}\right)^{k} u\right\rangle_{5-k, \mu, t}^{2}\right\} \\
\leqq C\left\{\|u(0)\|\left\|_{5, \mu}^{2}+\frac{1}{\mu}\right\| f \|_{4, \mu, t}^{2}+\frac{1}{\mu}\left\langle\Lambda_{y, \mu}^{1 / 2} g_{1}\right\rangle_{4, \mu, t}^{2}+\frac{1}{\mu}\left\langle\Lambda_{x, \mu}^{1 / 2} g_{2}\right\rangle_{4, \mu, t}^{2}\right\} .
\end{array}
$$

Proof. By the same method in [1: $\S 5]$, we have Lemma 6.1. Q.E.D.

Proof of Theorem 2. By Lemma 6.1 and the same arguments in [5: $\S 5]$, we have Theorem 2.

Q.E.D.

Proof of Theorem 4. We consider the mixed problem

$$
\left\{\begin{array}{l}
L_{1}\left[w_{1}\right]=\widetilde{f}(t, x, y) \\
w_{1}(0, x, y)=\widetilde{u}_{0}(x, y), \quad w_{1 t}(0, x, y)=\widetilde{u}_{1}(x, y) \\
\left.B_{4}\left[w_{1}\right]\right|_{y=0}=\widetilde{g}_{2}(t, x) \\
(t, x, y) \in \boldsymbol{R}_{+}^{1} \times \boldsymbol{R}^{1} \times \boldsymbol{R}_{+}^{1}
\end{array}\right.
$$

where $\tilde{l}$ is an extended function in the domain $\{(t, x, y) \mid t \geqq 0, x<0, y \geqq 0\}$ or $\{(x, y) \mid x<0, y \geqq 0\}$. Then, we have the solution $w_{1} \in \mathscr{H}_{8, \mu}\left[\boldsymbol{R}_{+}^{1} \times \boldsymbol{R}^{1} \times \boldsymbol{R}_{+}^{1}\right]$ of the problem (6.3) and $w_{1}$ has a compact support in the domain $\boldsymbol{R}_{x}^{1} \times \overrightarrow{\boldsymbol{R}}_{+y}^{1}$ for fixed $t(\geqq 0)$. We set

$$
n(t, y)=\left(\frac{\partial}{\partial y}+\beta\right) g_{1}(t, y)-\left.\left(\frac{\partial}{\partial y}+\beta\right)\left[\left(\frac{\partial}{\partial x}+\alpha\right) w_{1}\right]\right|_{x=0} .
$$

Then, we obtain, by $\left(I_{1}\right)$,

$$
\begin{aligned}
n(t, 0) & =\left(\frac{\partial}{\partial y}+\beta\right) g_{1}(t, 0)-\left.\left(\frac{\partial}{\partial x}+\alpha\right)\left[\left.\left(\frac{\partial}{\partial y}+\beta\right) w_{1}\right|_{y=0}\right]\right|_{x=0} \\
& =\left(\frac{\partial}{\partial y}+\beta\right) g_{1}(t, 0)-\left(\frac{\partial}{\partial x}+\alpha\right) g_{2}(t, 0)=0 .
\end{aligned}
$$

Also, by $\left(I_{3}\right)$ and $\left(I_{6}\right)$, we have

$$
\left\{\begin{array}{l}
n_{y y}(t, 0)=0 \\
n_{y y y y}(t, 0)=0
\end{array} .\right.
$$

We extend $n(t, y)$ to the region $\{y \mid y<0\}$ by the following

$$
\tilde{n}(t, y)=\left\{\begin{array}{cc}
n(t, y) & (y \geqq 0) \\
-n(t,-y) & (y<0)
\end{array}\right.
$$


Then, we have $\Lambda_{y, \mu}^{1 / 2} \tilde{n} \in \mathscr{H}_{b, \mu}\left[\left(\boldsymbol{R}_{+}^{1} \times \boldsymbol{R}^{1}\right)\right]$ and $\tilde{n}$ has a compact support in $\boldsymbol{R}_{y}^{1}$ for fixed $t(\geqq 0)$. Here, we consider the problem

$$
\left\{\begin{array}{l}
L_{1}\left[w_{2}\right]=0 \\
w_{2}(0, x, y)=0, \quad w_{2 t}(0, x, y)=0 \\
B_{3}\left[w_{2}\right]_{x=0}=\widetilde{n} \\
(t, x, y) \in\left(R_{+}^{1}\right)^{2} \times R^{1}
\end{array}\right.
$$

Then, we have the solution $w_{2}$ of the problem (6.7) which belongs to $\mathscr{H}_{8, \mu}\left[\left(\boldsymbol{R}_{+}^{1}\right)^{2} \times \boldsymbol{R}^{1}\right]$ and has a compact support in the region $\overline{\boldsymbol{R}}_{+\infty}^{1} \times \boldsymbol{R}_{y}^{1}$ for fixed $t(\geqq 0)$. Also, we have $w_{2}(t, x, 0)=0$. Next, we solve the equation

$$
\frac{\partial w_{8}}{\partial y}+\beta w_{8}=w_{2}
$$

for $L\left(\boldsymbol{R}_{+y}^{1}\right)$ space. Then, we have the solution

$$
w_{3}=e^{-\beta y} \int_{\infty}^{y} e^{\beta s} w_{2}(t, x, s) d s .
$$

We set

$$
u=w_{1}+w_{3} \text {. }
$$

By the above construction, we obtain the solution $u$ of the problem (II) which satisfies Theorem 4 .

Q.E.D.

§ 7. The existence of the solution (II).

In this section, we shall prove Theorem 6.

By the assumption, we extend $u_{0}, u_{1}, f, g_{1}$ and $g_{2}$ to the region $\{z \mid z<0\}$. We consider the problem

$$
\left\{\begin{array}{l}
L_{2}\left[w_{1}\right]=\widetilde{f}(t, x, y, z) \\
w_{1}(0, x, y, z)=\widetilde{u}_{0}(x, y, z), \quad w_{1 t}(0, x, y, z)=\widetilde{u}_{1}(x, y, z) \\
\left.B_{8}\left[w_{1}\right]\right|_{x=0}=\widetilde{g}_{1}(t, y, z) \\
\left.B_{6}\left[w_{1}\right]\right|_{y=0}=\widetilde{g}_{2}(t, x, z) \\
(t, x, y, z) \in\left(\boldsymbol{R}_{+}^{1}\right)^{8} \times R^{1}
\end{array}\right.
$$

By the assumption and the result in $\S 6$, we have the solution $w_{1} \epsilon$ $\mathscr{\mathcal { H }}_{10, \mu}\left[\left(\boldsymbol{R}_{+}^{1}\right)^{8} \times \boldsymbol{R}^{1}\right]$ of the problem (7.1) and $w_{1}$ has a compact support in the domain $\overline{\boldsymbol{R}}_{+x}^{1} \times \overline{\boldsymbol{R}}_{+y}^{1} \times \boldsymbol{R}_{z}^{1}$ for fixed $t(\geqq 0)$. We set

$$
n(t, x, y)=\left(\frac{\partial}{\partial x}+\alpha\right)\left(\frac{\partial}{\partial y}+\beta\right) g_{3}-\left.\left(\frac{\partial}{\partial x}+\alpha\right)\left(\frac{\partial}{\partial y}+\beta\right)\left(\frac{\partial}{\partial z}+\gamma\right) w_{1}\right|_{s=0} .
$$


Then, we have, by $\left(\mathrm{III}_{1}\right)$ and $\left(\mathrm{III}_{3}\right)$,

$$
\begin{aligned}
n(t, 0, y) & =\left(\frac{\partial}{\partial y}+\beta\right)\left\{\left[\left.\left(\frac{\partial}{\partial x}+\alpha\right) g_{3}\right|_{x=0}\right]-\left.\left(\frac{\partial}{\partial z}+\gamma\right)\left[\left.\left(\frac{\partial}{\partial x}+\alpha\right) w_{1}\right|_{x=0}\right]\right|_{z=0}\right\} \\
& =\left(\frac{\partial}{\partial y}+\beta\right)\left\{\left.\left(\frac{\partial}{\partial x}+\alpha\right) g_{3}\right|_{x=0}-\left.\left(\frac{\partial}{\partial z}+\gamma\right) g_{1}\right|_{z=0}\right\}=0
\end{aligned}
$$

and

$$
\begin{aligned}
n_{x x}(t, 0, y)= & \left(\frac{\partial}{\partial y}+\beta\right)\left\{\left[\left.\left(\frac{\partial}{\partial x}+\alpha\right) g_{s x x}\right|_{x=0}\right]-\left.\left(\frac{\partial}{\partial z}+\gamma\right)\left[\left.\left(\frac{\partial}{\partial x}+\alpha\right) w_{1 x x}\right|_{x=0}\right]\right|_{z=0}\right\} \\
= & \left(\frac{\partial}{\partial y}+\beta\right)\left\{\left.\left(\frac{\partial}{\partial x}+\alpha\right) g_{3 x x}\right|_{x=0}-\left(\frac{\partial}{\partial z}+\gamma\right)\left[\left(\frac{\partial^{2}}{\partial t^{2}}-\frac{\partial^{2}}{\partial y^{2}}-\frac{\partial^{2}}{\partial z^{2}}+d\right) g_{1}\right.\right. \\
& \left.\left.-\left.\left(\frac{\partial}{\partial x}+\alpha\right) f\right|_{x=0}\right]\left.\right|_{x=0}\right\} \\
= & 0 .
\end{aligned}
$$

Similarly, we get, by $\left(\mathrm{III}_{5}\right)$,

$$
n_{x x x x}(t, 0, y)=0
$$

Also, by $\left(\mathrm{II}_{1}\right),\left(\mathrm{II}_{3}\right)$ and $\left(\mathrm{II}_{3}\right)$, we obtain

$$
n(t, x, 0)=n_{y y}(t, x, 0)=n_{y y y y}(t, x, 0)=0 .
$$

Now, we consider the mixed problem

$$
\left\{\begin{array}{l}
L_{2}\left[w_{2}\right]=0 \\
u_{2}(0, x, y, z)=0, \quad w_{2 t}(0, x, y, z)=0 \\
\left.B_{7}\left[w_{2}\right]\right|_{z=0}=\tilde{n}(t, x, y) \\
(t, x, y, z) \in \boldsymbol{R}_{+}^{1} \times\left(\boldsymbol{R}^{1}\right)^{2} \times \boldsymbol{R}_{+}^{1}
\end{array}\right.
$$

where

$$
\tilde{n}(t, x, y)=\left\{\begin{array}{cc}
n(t, x, y) & (x \geqq 0, y \geqq 0) \\
-n(t,-x, y) & (x<0, y \geqq 0) \\
-n(t, x,-y) & (x \geqq 0, y<0) \\
n(t,-x,-y) & (x<0, y<0)
\end{array}\right.
$$

Then, we have the solution $w_{2} \in \mathscr{H}_{s, \mu}\left[\boldsymbol{R}_{+}^{1} \times\left(\boldsymbol{R}^{1}\right)^{2} \times \boldsymbol{R}_{+}^{1}\right]$ of the problem (7.7) which satisfies

$$
w_{2}(t, 0, y, z)=w_{2}(t, x, 0, z)=0
$$

and has a compact support in $(x, y, z)$ for fixed $t(\geqq 0)$. We solve the 
equation

$$
\left(\frac{\partial}{\partial x}+\alpha\right)\left(\frac{\partial}{\partial y}+\beta\right) w_{3}=w_{2}
$$

for $L^{2}\left(\boldsymbol{R}_{+x}^{1} \times \boldsymbol{R}_{+y}^{1}\right)$ space. Then, we get the solution

$$
w_{3}=e^{-\alpha x-\beta v} \int_{\infty}^{x} \int_{\infty}^{y} e^{\alpha r+\beta s} w_{2}(t, r, s, z) d r d s
$$

The function $w_{3}$ satisfies

$$
\left\{\begin{array}{l}
w_{3}(0, x, y, z)=w_{3 t}(0, x, y, z)=0 \\
\left.\left(\frac{\partial}{\partial x}+\alpha\right) w_{3}\right|_{x=0}=0 \\
\left.\left(\frac{\partial}{\partial y}+\beta\right) w_{3}\right|_{y=0}=0 \\
\left.\left(\frac{\partial}{\partial z}+\gamma\right) w_{3}\right|_{z=0}=-\left.\left(\frac{\partial}{\partial z}+\gamma\right) w_{1}\right|_{z=0}+g_{3}(t, x, y)
\end{array}\right.
$$

and

$$
L_{2}\left[w_{3}\right]=0
$$

We set $u=w_{1}+w_{3}$. By the above construction, we obtain the solution $u$ of the problem (III) which satisfies Theorem 6. Q.E.D.

\section{References}

[1] K. KojIma and M. TANIGUCHI, Mixed problem for hyperbolic equations in a domain with a corner, Funkcial. Ekvac., 23 (1980), 171-195.

[2] S. Miratake, Mixed problem for hyperbolic equations of second order with first order complex boundary operators, Japan. J. Math., 1 (1975), 111-158.

[3] S. MiYatake, A sharp form of the existence theorem for hyperbolic mixed problems of second order, J. Math. Kyoto Univ., 17 (1977), 199-223.

[4] M. TANIGUCHI, Mixed problem for wave equation in the domain with a corner, Funkcial, Ekvac., 21 (1978), 249-259.

[5] M. TANIGUCHI, Mixed problem for hyperbolic equations of second order in a domain with a corner, Tokyo J. Math., 5 (1982), 183-211.

[6] M. TANIGUCHI, Mixed problem for weakly hyperbolic equations of second order with degenerate first order boundary condition, Tokyo J. Math., 7 (1984), 61-98.

[7] M. TANIGUCHI, Mixed problem for weakly hyperbolic equations of second order with degenerate Neumann boundary condition, Funkcial. Ekvac., 27 (1984), 331-366. 